\title{
Dispersion of Response Times Reveals Cognitive Dynamics
}

\author{
John G. Holden \\ California State University, Northridge
}

\author{
Guy C. Van Orden \\ University of Cincinnati
}

\author{
Michael T. Turvey \\ University of Connecticut and Haskins Laboratories
}

\begin{abstract}
Trial-to-trial variation in word-pronunciation times exhibits $1 /$ f scaling. One explanation is that human performances are consequent on multiplicative interactions among interdependent processes-interaction dominant dynamics. This article describes simulated distributions of pronunciation times in a further test for multiplicative interactions and interdependence. Individual participant distributions of $\approx 1,100$ word-pronunciation times were successfully mimicked for each participant in combinations of lognormal and power-law behavior. Successful hazard function simulations generalized these results to establish interaction dominant dynamics, in contrast with component dominant dynamics, as a likely mechanism for cognitive activity.
\end{abstract}

Keywords: response time distributions, power laws, self-organization, word naming, 1/f noise

Cognitive studies of response times almost always concern components of cognitive architecture. This article is not about cognitive architecture. Instead, it poses a complementary question: How do the essential processes of cognitive activities interact to give rise to cognitive performance? The shapes of response time distributions supply information about how a system's processes interact. The response time data used to answer this question come from speeded naming of individual printed words. The characteristic shapes of pronunciation-time distributions (and their hazard functions) reveal the kind of interactions generally in cognitive activity.

This is not a new idea, and a familiar precedent exists for the logical inferences that are required. Familiar Gaussian distributions also support conclusions about how components interact, without requiring first that one know the identities, or details, of interacting components. This logic is introduced next using the example of skilled target shooting. Ballistics research was historically very important in the discovery and characterization of

John G. Holden, Department of Psychology, California State University, Northridge; Guy C. Van Orden, Center for Cognition, Action \& Perception, Department of Psychology, University of Cincinnati; Michael T. Turvey, Center for the Ecological Study of Perception and Action, University of Connecticut, and Haskins Laboratories, New Haven, Connecticut.

We acknowledge financial support from National Science Foundation Grants BCS-0446813 to John G. Holden and BCS-0642718 to John G. Holden and Guy C. Van Orden and from National Institute of Child Health and Human Development Grant HD-01994 to Haskins Laboratories.

We thank Greg Ashby, Jerald Balakrishnan, Ray Gibbs, David Gilden, Andrew Heathcote, Chris Kello, Howard Lee, Evan Palmer, and Trish Van Zandt for comments and other help with drafts of the manuscript.

Correspondence concerning this article should be addressed to John G. Holden, Department of Psychology, California State University, Northridge, 18111 Nordhoff Street, Northridge, CA 91330-8255. E-mail: jay.holden@csun.edu
Gaussian distributions. The explanation is somewhat tutorial, however, and readers who are familiar with how dispersion informs system theories and with the properties of lognormal and powerlaw distributions could skip forward to the section on the cocktail hypothesis.

\section{Dispersion and Interactions Among System Components}

A skilled target shooter's bullets form a familiar bell-shaped pattern around a bull's-eye. Strikes mostly pile up close to the bull's-eye. Moving further from the bull's-eye, on any radius, fewer and fewer strikes are observed. This pattern of bullet strikes is distributed as a bivariate Gaussian distribution, a fact worked out in late 19 th century and early 20 th century ballistics research. The Gaussian provides a relatively complete statistical description of how the bullet strikes are distributed.

Theoretical conclusions about the idealized bivariate Gaussian distribution explain how separate components of the targetshooting system combine to distribute bullet strikes in the Gaussian pattern. The dispersion or variability around the bull's-eye is the product of small accidental differences from shot to shot in the shape of the shell, the shell casing, and the amount of gunpowder in the shell, plus other innumerable weak, idiosyncratic, independently acting factors or perturbations (Gnedenko \& Khinchin, 1962). Each perturbation influences the trajectory of the projectile, if ever so slightly, on any given discharge of the weapon. All these independent causes of deviation add up to determine where the shooter's bullet will strike, the measure of distance from the bull's-eye.

Prior to this corroboration in ballistics, Laplace had worked out the relation between additive interactions and dispersion of measurements. Laplace reasoned that each measurement reflects the sum of many sources of deviation. This reasoning about dispersion of measurements allowed him to apply his central limit theorem. From that, he deduced that the overall distribution of measurements would appear in a bell shape, a curve that had been recently 
described by Gauss and, prior to that, by De Moivre (Porter, 1986; Stigler, 1986; Tankard, 1984).

Laplace's reasoning anchored Gauss's curve in explicit assumptions about the system being measured and how components interact to yield dispersion in the bell shape. These abstract assumptions were justified empirically in the later application to ballistics. Ballistics research effectively proved that large numbers of perturbations sum up their effects in ballistic trajectories to produce the Gaussian pattern (Klein, 1997). By the beginning of the 20th century, the abstract Gaussian description was trusted to describe the dispersion of measurements of almost any system (Stigler, 1986).

Gaussian distributions come from systems whose behavioral outcomes are subject to vast arrays of relatively weak, additive, and independently acting perturbations. Weak interactions among causal components ensure that perturbations affect components locally, individually, which allows effects to be localized in individual components. Weak interactions thus ensure component dominant dynamics because the dynamics within components dominate interactions among components.

Given the inherent links between additivity and research methods to identify causal components (cf. Lewontin, 1974; Sternberg, 1969), the question of how components interact arguably has priority over identifying components themselves. At least, one would want to establish first that components interact additively before applying the general linear (additive) model to infer components in component effects. If components interact in some way other than additive, then scientists require research methods appropriate to the other kind of interaction-at least, that is our contention (Riley \& Turvey, 2002; Riley \& Van Orden, 2005; Speelman \& Kirshner, 2005; Van Orden, Kello, \& Holden, in press; Van Orden, Pennington, \& Stone, 2001).

Of course, one could always mimic evidence of additive interactions in ways that do not truly entail weak additive interactions and component dominant dynamics, by artfully mimicking the Gaussian shape, for example. No theoretical conclusion is ironclad. Consider, on the one hand, even strongly nonlinear and discontinuous trajectories can be mimicked in carefully chosen component behaviors, combined additively. On the other hand, linear behaviors are not rare, even for systems of strongly nonlinear equations, which can make the term nonlinear dynamics appear superficially like an oxymoron.

Still, we accept assumptions that predominate empirically and provide simple yet comprehensive accounts, while we trust that scientific investigation, over the long term, will root out false assumptions. For example, the association between Gaussian patterns of dispersion and component dominant dynamics became so useful, so engrained, and so trusted, as to license the logical inverse of Laplace's central limit theorem. Thus, when an empirical distribution appears Gaussian, then the system must be subject to vast arrays of relatively weak, independently acting perturbations because it produced a Gaussian distribution. The Gaussian inference trusts empirical patterns of dispersion to reveal intrinsic dynamics as component dominant dynamics.

A reliable Gaussian account includes trustworthy links between ideal description, empirical pattern, and intrinsic dynamics of the system in question-how the components of a system interact. The account moves beyond superficial description to become a reliable systems theory. Understandably, early social scientists like
Galton and Pearson looked to this reliable theory for inspiration (Depew \& Weber, 1997; Klein, 1997; Porter, 1986; Stigler, 1986). The crucial point for the present article is that one can know how processes interact without knowing what these processes are. The necessary information is found in the abstract shape of dispersion alone.

\section{Multiplicative Interactions and Lognormal Dispersion}

At the beginning of the 21 st century, other abstract distributions of measured values vie for scientists' attention. Many physical, chemical, and biological laws rely on multiplicative operations, for instance, which yield a lognormal pattern of dispersion in measurements (Furusawa, Suzuki, Kashiwagi, Yomo, \& Kaneko, 2005; Limpert, Stahel, \& Abbt, 2001). We stay with the metaphor of ballistics to explain how multiplicative interactions deviate from the additive Gaussian pattern.

Imagine a slow-motion film that tracks a bullet's trajectory from the point the bullet exits a rifle's muzzle to its entry point at the target. At any point along its trajectory, the bullet's location on the next frame of film, after the next interval of time, can be predicted by adding up the effects of all the independent perturbations that acted on the bullet, up to and including the present frame.

Sources of variation are independent of each other. Some push left, some right, some up, some down, and most push in oblique directions, but the overall effect is represented simply by their sum. Large deviations from the bull's-eye are uncommon, for a skilled shooter at least, and a sufficient number of bullet strikes will yield the expected Gaussian pattern.

Imagine next a magic bullet for which sources of variation combine multiplicatively. This violates the Newtonian mechanics of bullets, of course, but we pretend that our magic bullet exists. Each change in the magic bullet's trajectory is the product of the current trajectory and the force of the perturbation.

For magic bullets, sometimes, multiplication has a corrective influence and shrinks the effects of previous perturbations, when the multiplier is greater than zero but less than one. Other times, the multiplier amplifies previous perturbations and exaggerates the dispersion that they cause, when the multiplier is greater than one.

For magic bullets, corrective multiplicative interactions tend to concentrate bullets already close to the bull's-eye in a tighter distribution, while amplifier multiplications produce more extreme deviations away from the bull's-eye. Multiplication erodes the middle range of deviation compared with addition. It produces a dispersion of bullets that is denser at its center and more stretched out at the extreme.

The result, in the limit, appears as a lognormal distribution. The name comes from the fact that a lognormal will reappear as a standard Gaussian or normal distribution if its axis of measurement undergoes a logarithmic transformation. The transformation redistributes the variability to become once again a symmetric Gaussian distribution, as a comparison of Figures 1A and 1B illustrates.

Gaussian and lognormal patterns are closely related. As such, they share the assumption that variation comes from perturbations of independent processes. Multiplication in the linear domain equals addition in the logarithmic domain. If vast arrays of independent perturbations interact as in a serial chain of multiplications, then event times such as response times will be lognormally distributed. Independent processes also imply that both distribu- 

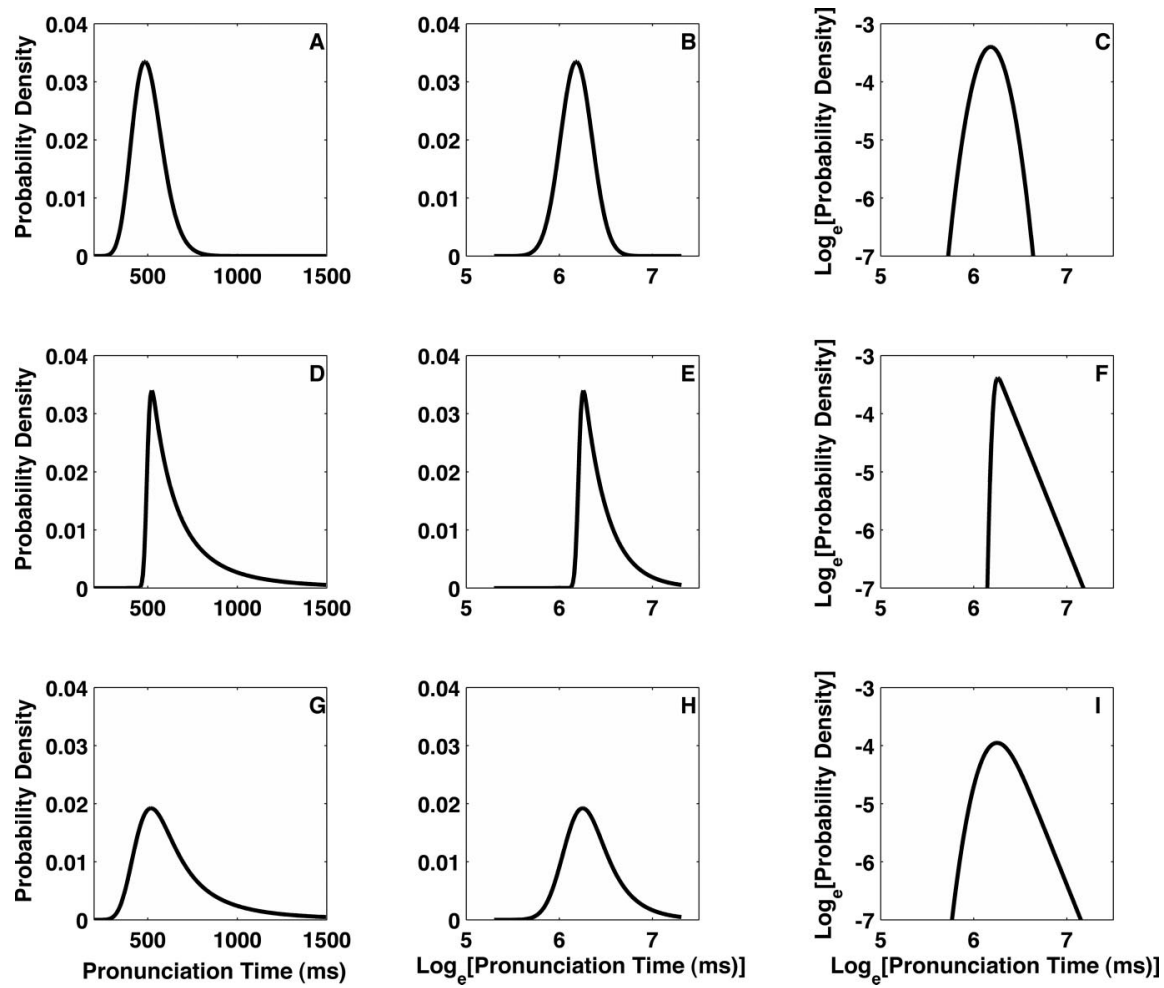

Figure 1. A: An ideal lognormal probability density function, plotted on standard linear axes. The $x$-axis depicts response time, and the $y$-axis tracks the probability of observing a response time in any given interval of time. B: The same density, now on log-linear axes, where it appears as a symmetric, standard Gaussian density. C: The same density curve, now plotted on double-logarithmic axes, where it appears as a downward turning parabola. D: An ideal inverse power-law density on standard linear axes. E: The same power-law density on log-linear axes. Notice that the power-law density maintains its extreme, slowly decaying tail distinct from a simple exponential-tailed density, which falls off linearly on log-linear scales. F: The power-law density falls off as a line on double-logarithmic axes, the characteristic footprint of a power-law scaling relation. G: The density depicts an idealized $50 \%-50 \%$ cocktail of the two density functions on standard linear axes. H: The same mixture density in log-linear domain. I: The same mixture density in the double-logarithmic domain. Depicting the probability density functions on the different scales helps to distinguish them from other potential ideal descriptions.

tions are additive, one on a linear scale and the other on a logarithmic scale.

Yet lognormal distributions are produced by multiplicative interactions, which also makes them cousins to multiplicative interdependent interactions. Multiplicative interdependent interactions produce power laws. As kin to power laws, systems that produce lognormal distributions illustrate a stable, special case of interdependence. Lognormal dispersion is found in multiplicative feedback systems for which the interacting processes are sufficiently constrained (Farmer, 1990). Sufficiently constrained interactions quickly stabilize solutions, minimizing the empirical consequences of feedback, and give the impression of independent processes. Next, we discuss multiplicative feedback more generally and its consequences for dispersion of measurements.

\section{Multiplicative Feedback Interactions and Power-Law Dispersion}

Aggregate event times of interdependent processes accumulate in lawful patterns called power laws. Specifically, in the aggregate, interdependent processes produce response times, or event times, in the pattern of an inverse power law-a straight line on an $x$-axis of $(\log )$ magnitude and a $y$-axis of $(\log )$ frequency of occurrence. For example, fast response times are relatively common, and very slow response times are rare. If the pattern is a power law, however, then the frequency of occurrence of a particular response time will be directly related to its magnitude.

The probability of a particular event time equals beta times that event time raised to the power of negative alpha. Alpha is a scaling exponent that describes the rate of decay in the slow tail of the distribution. Beta is a scaling term; it moves the equation up and down on the $y$-axis. (This is clearer in Equation 2, below, in which Equation 1 has been transformed by taking the logarithm of both sides.) The power-law relation is represented in Equations 1 and 2.

$$
\mathrm{P}(\text { event time })=\beta \times \text { event time } \mathrm{e}^{-\alpha} .
$$

$$
\log [\mathrm{P}(\text { event time })]=-\alpha \times \log (\text { event time })+\log (\beta) .
$$


Notice that Equation 2 is simply the equation for a line; the (log) probability of an event time equals negative alpha (slope) times the $(\log )$ event time itself plus a constant $(\log [\beta])$. The graph of an ideal inverse power law is simply a line with a negative slope on double-logarithmic axes (see Figure 1F). The key term in these equations is alpha. Alpha is the exponent of the power law and describes the rate of decay in the skewed tail of the distribution.

Equation 1 will diverge as event times approach zero. Consequently, raw variables are often normalized to make beta intercept the $y$-axis. Instead, in Figure 1, the graphic depiction of the power law appends a low-variability, lognormal front-end to close up the probability density. This allows the probability density function to be shifted off the $y$-intercept and to resemble standard probability density functions of response time.

The power-law Equations 1 and 2 describe possible patterns of dispersion in measurements. Recall that the dispersion of measurements in ballistics is due to a vast array of independent perturbations, of bullet casings, powder load, and so on. Interdependent interactions are likewise subject to vast arrays of perturbations, amplified in recurrent multiplicative interactions due to feedback. Feedback behavior is also lawful behavior in this case, and the dispersion of $(\log )$ event times remains proportional to the magnitudes of the perturbations.

Many natural systems yield event magnitudes that obey inverse power laws, and power laws are associated with a wide array of organisms, biological processes, and collective social activities (Bak, 1996; Farmer \& Geanakoplos, 2005; Jensen, 1998; Jones, 2002; Mitzenmacher, 2003; Philippe, 2000; West \& Deering, 1995). Allometric laws are examples of power-law scaling in biology, although they are not statistical distributions like the topic of this article. Power-law dispersion most like response time dispersion includes Zipf's law, earthquake magnitudes, book and online music sales, and scientific citation rates (Anderson, 2006; Turvey \& Moreno, 2006). These are all succinctly described as inverse power-law distributions.

Anderson (2006) and Newman (2005) include more examples of power-law behavior. Newman (2005) and Clauset, Shalizi, and Newman (2007) are good sources for mathematical and statistical details of power laws. The key to understanding power-law behavior is amplification via multiplicative feedback, to which we return several times in this article.

The feedback interactions that produce power-law behavior are called interaction dominant dynamics (Jensen, 1998). Feedback spreads the impact of perturbations among interacting components. Consequently, one can no longer perturb individual components to produce isolated effects. Multiplicative feedback creates stronger interactions among components and distributes perturbations throughout the network of components. It is this property of interaction dominant dynamics that promotes a global response to perturbations in systems that organize their behavior in multiplicative feedback.

\section{The Cocktail Hypothesis}

The previous sections introduced power-law and lognormal distributions. Cognitive dynamics are multiplicative if response time distributions entail either or both of these distributions. Cognitive dynamics are interdependent if response times are distrib- uted as power laws. Multiplicative interdependent dynamics are interaction dominant dynamics, as noted in the previous paragraph.

Interaction dominant dynamics ensure necessary flexibility in cognition and behavior (Warren, 2006). Flexibility is achieved when interaction dominant dynamics self-organize to stay near choice points, called critical points, which separate the available options for cognition and behavior, thus the technical term selforganized criticality (Van Orden, Holden, \& Turvey, 2003). Interaction dominant dynamics anticipated widely evident fractal $1 / \mathrm{f}$ scaling, found in trial series of response times and other data (Gilden, 2001; Kello, Anderson, Holden, \& Van Orden, 2008; Kello, Beltz, Holden, \& Van Orden, 2007; Riley \& Turvey, 2002).

This article is not about $1 / \mathrm{f}$ scaling, however. The predictions tested here simply derive from the shared parent hypothesis, interaction dominant dynamics, which predicts fractal 1/f scaling. The shared parent strictly limits choices for possible distributions of response times. Most famously and straightforwardly, it favors data distributed as an inverse power law relating the magnitude ( $x$-axis) and likelihood ( $y$-axis) of data values (Bak, 1996).

Of course, response times are not exclusively power laws, thus the hypothesis of lognormal behavior for the fast front end. Lognormal behavior is a motivated hypothesis due to its theoretical relation to power-law behavior. The theoretical explanation can be found in West and Deering (1995), for example, who placed power-law and lognormal behavior on a continuum with Gaussian distributions (see also Montroll \& Shlesinger, 1982).

The Gaussian, a signature of weak additive interactions among independent, random variables-component dominant dynamics - is at one end of the continuum. At the other extreme is the inverse power law, a signature of interdependent multiplicative interactions-interaction dominant dynamics. The lognormal stands between the two extremes because it combines independent, random variables with multiplicative interactions.

In our turn, we have added one fact to the above: Power-law behavior transitions to lognormal behavior if sufficient constraints accrue to mask superficial consequences of feedback. For example, this is observed in generic recurrent neural models in which available constraints include configurations of connection weights (Farmer, 1990). More generally, available constraints derive from history, context, the current status of mind and body, the task at hand, and their entailments - available aspects of mind, body, and world that reduce or constrain the degrees of freedom for cognition and behavior (Hollis, Kloos, \& Van Orden, 2009; Kugler \& Turvey, 1987).

Notice how constraints naturally motivate new predictions about mixtures of power-law and lognormal behaviors and about the direction of change in relative mixtures as interactions become more or less constrained, due to practice or rehearsal, for example (cf. Wijnants, Bosman, Hasselman, Cox, \& Van Orden, 2009), or aging, damage, and illness (cf. Colangelo, Holden, Buchanan, \& Van Orden, 2004; Moreno, Buchanan, \& Van Orden, 2002; Van Orden, 2007; West, 2006). Available constraints determine the mixture of power-law and lognormal dispersion, which invites analyses that may reject either lognormal, power law, or the mixture of both in response time dispersion.

Figure 1 summarizes the ideal patterns of dispersion on linear, log-linear, and log-log axes (see caption). Figure 1 also illustrates a cocktail mix of power-law and lognormal dispersion. Each pronunciation time is sampled from either a power law or a 
lognormal that, in the aggregate, makes a power-law-lognormal cocktail. We call this a cocktail because each word's pronunciation time, like a liquid molecule in a cocktail, comes from separate power-law or lognormal bottles. In the subsequent cocktail, response times are mixed in proportions that pile up as a participant's aggregate distribution. The proportion of power-law behavior and the exponent of the power law are the key parameters of this hypothesis.

Like a cocktail, different collections of response times, from different participants, from different task conditions, from the same participant on different tasks, or from the same task on different occasions, can be a different mix of power-law and lognormal behavior. The mix proportions can range from predominantly power-law behavior to almost exclusively lognormallognormal straight up with a dash of power law, so to speak (compare Holden, 2002; Van Orden, Moreno, \& Holden, 2003).

Notice that each response time could summarize deterministic, stochastic, or random component contributions or all of the above. The cocktail hypothesis generalizes across all these cases because we make assumptions not about the intrinsic dynamics of components, only about how they interact. The total event time combines all factors in multiplicative interactions yielding a response time that is either a power-law or lognormal sample.

\section{The Hazard Function Test}

We now introduce a test for generality of the cocktail hypothesis. In the early chapters of Luce's (1986) classic survey of response time studies, he underscored the importance of reconciling cognitive theory with the widely identified characteristic shapes of response time hazard functions. Hazard functions are mathematical transformations of probability density functions and their cumulative distributions. They portray information so that new questions can be asked about the probability of events. For instance, the hazard function portrait answers the question, What is the likelihood that an event will occur now given that it has not occurred so far? We describe more details of hazard functions later, but here, we focus on how they function as a test of generality.

Three characteristic shapes are found for hazard functions across otherwise different laboratory tasks and manipulations. The three shapes emerge in single experiments designed to examine a single kind of performance, such as word pronunciation. A risk of paradox exists because the three shapes could indicate qualitatively different dynamics (Luce, 1986); such heterogeneity could require that analyses refocus on details of intraindividual variation, for instance (Molenaar, 2008).

Consequently, although hazard functions are typically ignored in response time studies, they are nonetheless sources of limiting constraints for theories of response time (Ashby, Tien, \& Balakrishnan, 1993; Balakrishnan \& Ashby, 1992; Maddox, Ashby, \& Gottlob, 1998). Arguably, characteristic hazard functions offer critical linchpins between data and theory because they are so difficult to simulate with limited ad hoc assumptions (Luce, 1986; Maddox et al., 1998; Van Zandt \& Ratcliff, 1995).

It is this fact, that they are not easily mimicked, that makes hazard functions useful to test generality. For instance, all other things being equal, to mimic the full shape of a probability density function is to provide a more complete account of response times, compared with accounts focused on summary statistics like means or standard deviations. In the same vein, to successfully mimic a hazard function also expands the inclusiveness, completeness, and generality of an account. Townsend (1990) used these facts to argue why the qualitative ordering of hazard functions is more diagnostic than an ordering of condition means or even probability density functions, for example.

Thus, in the present case, all other things being equal, if mathematical transformations of simulated density functions yield the same hazard functions as their empirical counterparts, then the simulations have met a much greater challenge than density functions alone. Generality accrues in meeting this challenge because one can successfully mimic empirical density functions, for example, and still fail to mimic their derived hazard functions, but not vice versa. Consequently, in the existence proofs that follow, we fit individual participants' density functions of word-naming times and also fit participants' hazard functions.

As the simulations reveal, one easily recognizes in the cocktail simulations the characteristic hazard functions that other cognitive scientists have so carefully excavated. Luce (1986) and Maddox et al. (1998) described the three characteristic empirical hazard functions of response time distributions: Either the hazard function rises monotonically to an asymptote (compare Figure $2 \mathrm{G}$ ), or it rises rapidly to a peak and then declines to an asymptote (see Figure $2 \mathrm{H}$ ), or it rises rapidly to a much higher peak and then falls off sharply (see Figure 2I). This remarkably succinct characterization of response time outcomes in very many or all cognitive tasks is a basis for another kind of generality. A theory of response times that explains the three characteristic hazard functions generalizes to response times at large.

\section{An Existence Proof Using Word-Pronunciation Times}

We begin with an existing data set of word-pronunciation times (Thornton \& Gilden, 2005; Van Orden, Holden, \& Turvey, 2003). A word-pronunciation trial presents the participant with a single word that he or she pronounces aloud quickly. Pronunciation time is measured from when the word appears until the participant's voice triggers a voice relay. The data come from a wordpronunciation experiment in which 1,100 pronunciation trials presented four- and five-letter monosyllabic words in a unique random order, across trials, to each of 20 participants, one word per trial. Empirical power laws can only be distinguished in the slow extremes of rare response times, and large samples of withinparticipant response times better fill out the slow extremes of a person's response time distribution.

\section{Simulations of Participant Probability Density Functions}

Figures 2A, 2B, and 2C depict 3 individual participants' pronunciation-time probability density functions. The smooth and continuous functions are products of a standard procedure of nonparametric, lognormal-kernel smoothing (Silverman, 1989; Van Zandt, 2000; a lognormal kernel is equivalent to a Gaussian kernel in the log-linear domain). Construction of density functions, including smoothing, was done after a logarithmic transformation of raw pronunciation times. The three figures illustrate the three categories of distributions that produce characteristic hazard function shapes. We explain more details of hazard functions after 

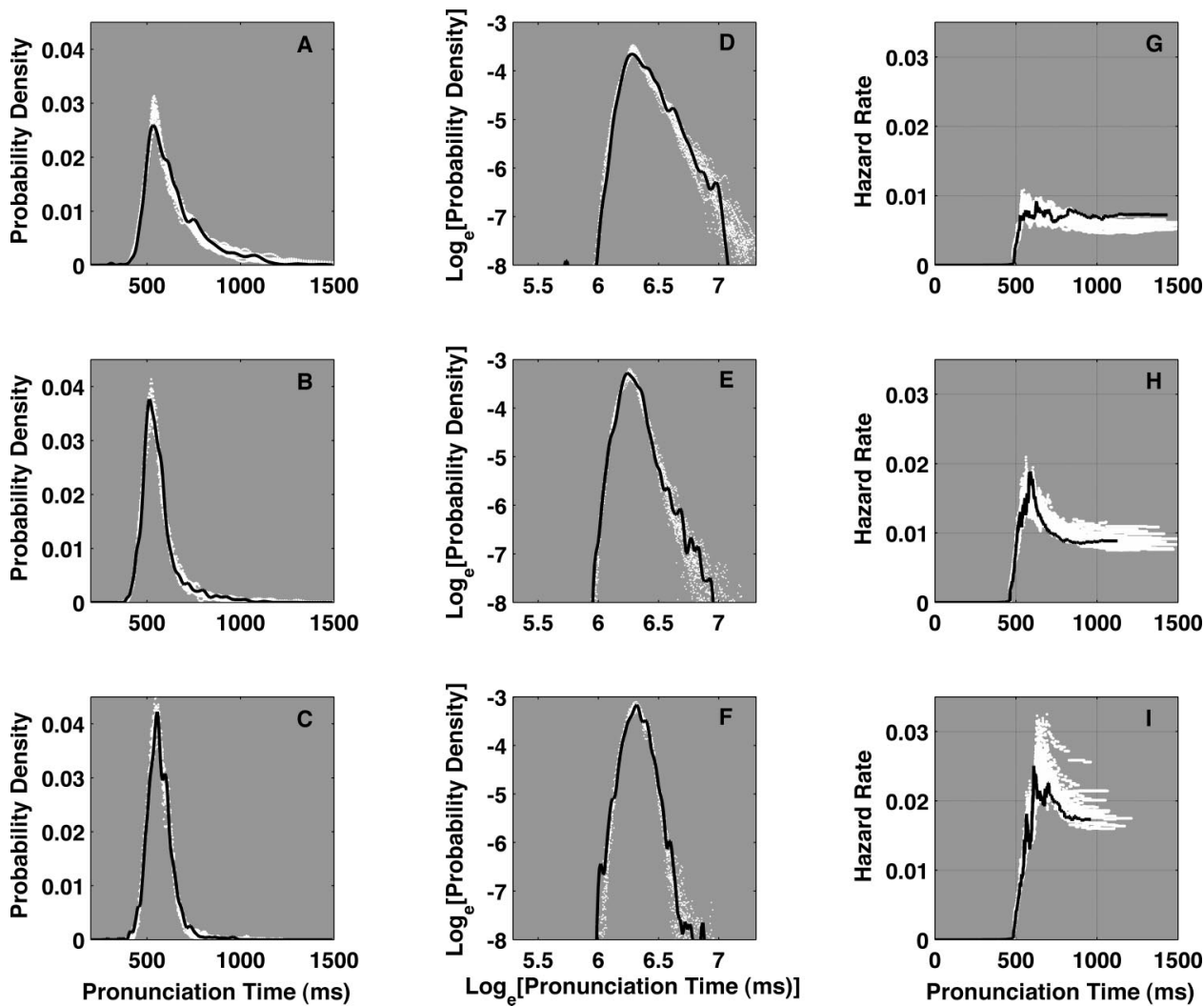

Figure 2. Three characteristic hazard functions found generally in response time data and the distributions of pronunciation times from which they were computed, on linear (Panels A, B, and C) and log-log (Panels D, E, and F) scales. Panels A, B, and C correspond to pronunciation times of three individual participants from the first existence proof. The heavy black line in each panel is the probability density of one participant's pronunciationtime distribution. The white points surrounding each black line in each panel represent 22 simulation mixtures of ideal lognormal and inverse power-law distributions, using fixed parameters of synthetic distributions in a resampling or bootstrap technique (cf. Efron \& Tibshirani, 1993). The boundaries established by the white clouds of the 22 simulated distributions circumscribe a $90 \%$ confidence interval around each empirical probability density and hazard function.

describing the methods used to simulate individual participants' probability density functions.

\section{Simulation Methods}

Cocktail mixture simulations were conducted to capture each of the 20 shapes of empirical density curves from the 20 participants' data reported in Van Orden, Holden, and Turvey (2003). Simulations mixed together synthetic samples from parent distributions of inverse power-law and lognormal pronunciation times. As the simulations demonstrate, the sample mixtures sufficed to mimic participant data. Each choice of parameter values mimicked one participant's pronunciation-time distribution.

Data preparation. The criteria to select the data points to be simulated were conservatively inclusive. In a sample of the skilled word-naming literature, the most conservative criteria for exclusion were cutoffs less than $200 \mathrm{~ms}$ and greater than 1,500 ms (cf. Balota, Cortese, Sergent-Marshall, Spieler, \& Yap, 2004; Balota \& Spieler, 1999; Spieler \& Balota, 1997). We also included the small proportion of pronunciation times that resulted in errors because it did not change the outcome $(M=2.12 \%, S D=1.48 \%)$. Inclusion of error response times is also conservative; we assume errors are produced by the same dynamics that produce correct pronunciations.

The total number of simulated response times, from a participant's data set, equaled the total number of pronunciation times that were greater than or equal to $200 \mathrm{~ms}$ and less than or equal to $1,500 \mathrm{~ms}$. The maximum possible number was 1,100 . The actual number varied from participant to participant $(M=1,094, S D=$ 8.76). The number of empirical observations on this interval determined the number of synthetic data points generated in the same interval on each replication of a simulation.

Parameters. The cocktail simulations included seven parameters: the mode $\Omega_{\mathrm{PL}}$ and exponent $\alpha$ of the inverse power law, the lognormal mode $\Omega_{\mathrm{LN}}$ and standard deviation $\sigma$, the sample mixture proportions $\rho_{\mathrm{FLN}}$ and $\rho_{\mathrm{BLN}}$ that index relative proportions of lognormal behavior on each side of the lognormal mode, and $\rho_{\mathrm{PL}}$, which indexes the proportion of power-law behavior in the slow tail. Only two of the three proportion parameters were free to vary 
since the relative proportions must sum to one. Thus, six parameters were available to simulate individual patterns of dispersion across participants. By comparison, standard diffusion models that simulate decision time data collapsed across participants include at least seven parameters, and up to nine have been used in some circumstances (Wagenmakers, in press).

On log-response time axes, the mode $\Omega_{\mathrm{LN}}$ of a parent lognormal is also its midpoint. The standard deviation $\sigma$ controls relative width around this midpoint. The mode $\Omega_{\mathrm{PL}}$ of the parent power law is also its maximum probability and its starting point- that is, the minimum or fastest response time on the $x$-axis at which power-law behavior is inserted. On double-logarithmic axes, the exponent parameter $\alpha$ indexes the rate of linear decay of the slow power-law tail. On linear axes, the slow tail of the distribution diminishes as the curve of power-law decay (e.g., compare Figure $1 \mathrm{~F}$ with Figure 1D).

Choosing the mode parameters. Almost all parameter choices for synthetic parent distributions were equated with estimated parameters of participants' empirical distributions. For example, in most cases, the parent lognormal mode $\Omega_{\mathrm{LN}}$ was equal to the estimate of the mode of the target empirical distribution, with the following caveat: The mean, not the mode, is specified in equations that define the shape of a lognormal density. However, on log axes, the mean and mode are equal in the statistical long run, and the mode is relatively impervious to extreme values in the slow tail. Since the parameters of the lognormal densities were specified in the logarithmic domain, it was convenient to substitute the log of the empirical modes in these equations.

The value of the empirical mode was established using a bootstrap routine that repeatedly resampled and estimated the location of the empirical mode (Efron \& Tibshirani, 1993). Thirteen of the 20 participants' distributions were successfully approximated using lognormal mode parameters pulled directly from the mode statistics of the empirical distributions. Seven additional distributions required a slight adjustment of the statistical estimate of the mode to align the empirical and synthetic distributions.

Some empirical distributions appeared to be bimodal, which can be accommodated by setting the faster time mode equal to the lognormal mode $\Omega_{\mathrm{LN}}$ and the slower time mode equal to the power-law mode $\Omega_{\mathrm{PL}}$. Thirteen of the 20 empirical distributions were bimodal; the average difference between modes was $29 \mathrm{~ms}$. Apparent spurious bimodality was also present however (e.g., two modes very close to each other). Spurious versus real bimodality was judged from the hazard functions, where real bimodality has a more prominent effect. For 7 participants' simulations, the power-law mode $\Omega_{\mathrm{PL}}$ and lognormal mode $\Omega_{\mathrm{LN}}$ were equivalent.

Choosing the dispersion parameters. The seed estimate to find a standard deviation $(\sigma)$ for the parent lognormal came from a conventional error-minimization fitting routine. The routine fits a lognormal curve to the fast front curve of the empirical distribution, up to and including the empirical mode. Past this point, however, the standard deviation parameter $\sigma$ was adjusted by hand to improve the fit.

All synthetic pronunciation times less than or equal to an empirical distribution's (fastest) mode (the $\Omega_{\mathrm{LN}}$ parameter) were sampled exclusively from the lognormal parent. In every case, the proportion of synthetic lognormal data points was the same as the corresponding proportion of the participant's data points, less than or equal to the empirical mode. The parameter $\rho_{\mathrm{FLN}}$ indicates the proportion of synthetic times less than or equal to the mode $\Omega_{\mathrm{LN}}$.

The parameter $\rho_{\mathrm{BLN}}$ equals the maximum proportion of synthetic lognormal times greater than $\Omega_{\mathrm{LN}}$. The actual proportion of synthetic lognormal times greater than $\Omega_{\mathrm{LN}}$ depends on the tradeoff of lognormal and power-law behavior in the slow end of the simulated distribution. The slow tails of distributions were hand-fit using small adjustments to the two remaining free parametersnamely, the exponent parameter $\alpha$ and the proportion $\rho_{\mathrm{PL}}$ of power-law behavior in the slow-tail mixture. Synthetic mixtures were eyeballed and adjusted using a program that allowed visual comparison of synthetic and empirical density and hazard functions. The program required that all parameters were set to some value in any and all adjustments.

Synthetic lognormal-power-law mixture densities were realized in the following manner. First, both a lognormal and a power-law density function, defined according to specific mode and dispersion parameters, were generated and normalized to occupy unit area within the specified response time interval. The densities were then combined in the required proportions, on either side of the lognormal mode, according to a formula for generating mixture densities provided by Luce (1986, pp. 274-275). The equation for a normalized power-law density appeared in Clauset et al. (2007). The resulting cocktail density was then transformed to a cumulative distribution function. Following that, a rectangular unitinterval random number generator was used to produce the required number of synthetic samples from the inverse of the mixture distribution function.

Initially, the power-law exponent parameter $\alpha$ was set to a relatively small, shallow value. If initial attempts to mimic the empirical distribution failed across a range of mixture proportions, then the exponent of the parent power law was increased to a larger, steeper value, and another attempt was made. Trial-anderror fitting continued until an apparently optimal choice of parameters was reached to approximate the density (and hazard) function of a participant's data. Table 1 lists the parameter values of the parent distributions for each participant's simulated data.

Simulation details. With parameter values in place, each individual's pronunciation-time distribution was simulated 22 times, and one synthetic distribution was selected at random for a statistical contrast. The contrast was a two-sample KolmogorovSmirnov goodness-of-fit test with a Type I error rate of .05. Success was counted if the synthetic distribution captured the prominent features of the participant's density functions and if it passed the Kolmogorov-Smirnov test.

All 22 independent simulation outcomes were plotted in white, behind each participant's black empirical curve (see, e.g., Figure 2 ). The boundaries that define the cloud of white points circumscribe statistical estimates of the 5th and 95th percentiles around each empirical probability density and hazard function (Efron \& Tibshirani, 1993). Thus, the repeated replications of the synthetic distributions establish $90 \%$ confidence intervals around the empirical density and hazard functions. The fact that so few replications of the synthetic distributions so closely approximate the empirical curves makes plausible, in a statistical sense, that cocktail mixtures are reliable descriptions of the empirical patterns.

Shortly, we discuss details of three categories of simulated distributions, power-law dominant, intermediate mixtures, and lognormal dominant, right after we describe how we arrived at 
Table 1

Parameters Used to Generate Synthetic Distributions for the First Existence Proof

\begin{tabular}{|c|c|c|c|c|c|c|c|}
\hline Participant & $\Omega_{\mathrm{LN}}$ & $\sigma$ & $\Omega_{\mathrm{PL}}$ & $\alpha$ & $\rho_{\mathrm{FLN}}$ & $\rho_{\text {BLN }}$ & $\rho_{\mathrm{PL}}$ \\
\hline 1 & 6.2500 & .100 & 6.270 & 6.00 & .311 & .019 & .670 \\
\hline 2 & 6.2560 & .095 & 6.256 & 5.50 & .241 & .000 & .759 \\
\hline 3 & 6.3200 & .110 & 6.460 & 7.75 & .370 & .397 & .233 \\
\hline 4 & 6.1230 & .085 & 6.240 & 8.50 & .315 & .405 & .280 \\
\hline 5 & 6.2000 & .090 & 6.210 & 7.50 & .355 & .045 & .600 \\
\hline 6 & 6.3700 & .110 & 6.370 & 8.00 & .379 & .001 & .620 \\
\hline 7 & 6.2540 & .110 & 6.400 & 8.00 & .474 & .446 & .080 \\
\hline 8 & 6.1269 & .090 & 6.230 & 8.50 & .293 & .347 & .360 \\
\hline 9 & 6.2050 & .100 & 6.205 & 8.75 & .417 & .000 & .583 \\
\hline 10 & 6.3490 & .100 & 6.510 & 8.00 & .494 & .466 & .040 \\
\hline 11 & 6.2530 & .095 & 6.253 & 7.50 & .428 & .192 & .380 \\
\hline 12 & 6.2400 & .110 & 6.240 & 8.75 & .476 & .224 & .300 \\
\hline 13 & 6.2360 & .090 & 6.300 & 7.75 & .375 & .425 & .200 \\
\hline 14 & 6.1400 & .100 & 6.250 & 8.25 & .513 & .407 & .080 \\
\hline 15 & 6.1760 & .120 & 6.280 & 8.50 & .378 & .452 & .170 \\
\hline 16 & 6.2300 & .110 & 6.230 & 8.50 & .436 & .204 & .360 \\
\hline 17 & 6.2540 & .085 & 6.270 & 8.50 & .371 & .089 & .540 \\
\hline 18 & 6.1440 & .110 & 6.165 & 10.00 & .525 & .295 & .180 \\
\hline 19 & 6.0400 & .080 & 6.060 & 10.00 & .370 & .230 & .400 \\
\hline 20 & 6.3040 & .090 & 6.400 & 10.00 & .445 & .465 & .090 \\
\hline$M$ & 6.2200 & .100 & 6.280 & 8.21 & .398 & .255 & .346 \\
\hline
\end{tabular}

Note. This table lists parameters of the parent lognormal and power-law distributions, as well as the proportion of power-law samples used in the simulations. The participant numbers were established by ordering each distribution in terms of the empirically estimated slope of the tail of the distribution. This explains why the participant numbers correspond to a close rank ordering of the alpha parameter. Participants 1 and 2 were classified as power-law dominant; Participants 17-20 were classified as lognormal dominant. The remaining participants were classified as intermediate mixtures. The full collection of simulations can be viewed online at http://www.csun.edu/ jgh62212/RTD. $\Omega_{\mathrm{LN}}=$ lognormal mode; $\sigma=1$ lognormal standard deviation; $\Omega_{\mathrm{PL}}=$ power-law mode; $\alpha=$ power-law tail; $\rho_{\mathrm{FLN}}=$ proportion in front end of lognormal; $\rho_{\mathrm{BLN}}=$ proportion in back end of lognormal; $\rho_{\mathrm{PL}}=$ proportion power law.

those category distinctions. First, a line was fit to the slow tail of each participant's density, on log-log axes, beginning at the empirical mode. The slope of the line was then used to rank empirical distributions from most shallow to most steep. The distributions with the most shallow rank versus the most steep rank are what we eventually call power-law versus lognormal dominant distributions, respectively.

Of interest here is that the rank of distributions, according to estimates of unadulterated power-law behavior (their slow-tail exponents), also respected an ordering of the three characteristic hazard functions. Small unadulterated exponents corresponded to hazard functions that rose to relatively constant asymptotes and tended to lack prominent peaks. These required relatively higher proportions of power-law behavior drawn from distributions with smaller exponents.

By contrast, distributions with larger exponents corresponded to hazard functions that rapidly rose to a high peak and tended to require low proportions of power-law behavior from parent power laws with large exponents. The remaining distributions, between the two extremes, had hazard functions that rose to an intermediate peak and declined to an asymptote. These intermediate cases combine the features of the extreme cases. Simulations drew intermediate proportions of power-law behavior and/or drew from a power-law distribution with an intermediate exponent.

Nonetheless, the relation between hazard function shape and cocktail parameters is neither isomorphic nor monotonic. This is due partly to the log scales. More extreme linear values are more compressed on log scales. Consequently, a power law with a mode equaling $400 \mathrm{~ms}$ that decays with an exponent of 4 will cover a narrower range of linear values than a power-law distribution with the same exponent and a 600-ms mode, for example.

If one could create comparable modes on the response time axis, however, then the three categories of hazard functions could be set on a continuum that ties together proportion of power-law behavior and the magnitude of the power-law exponent $(\alpha)$. However, our goal was to simulate actual values of empirical distributions.

Except where noted, these same methods and criteria were applied in all simulations reported in this article. PDF files containing plots of all simulations are available online at http:// www.csun.edu/ jgh62212/RTD.

\section{Simulation Results}

Characteristic distributions. All participants' data came from a common set of stimulus words. Nevertheless, different participants produced visibly distinct distributions, as we illustrate in Figure 2. The obvious difference is in the relative skew of slow pronunciation-time tails (the fast lognormal front ends are pretty much the same shape). Figure $2 \mathrm{~A}$ includes a dramatically stretched slow tail. Figure 2B also has a visible positive skew, but less dramatic than in Figure 2A, while the density depicted in Figure $2 \mathrm{C}$ is similar to the lognormal density in Figure 1A. The solid black curves in Figures 2D, 2E, and $2 \mathrm{~F}$ illustrate the same 3 participants' density functions, now plotted on double-logarithmic axes. In each plot, the $x$-axis is the natural logarithm of pronunciation time, and the $y$-axis is the natural logarithm of the probability density. (Wavy oscillations in extreme tails are an artifact of the sparse observations in the extreme tail.) 
Power-law dominant. Figure 2A shows synthetic density functions plotted as white points behind the participant's empirical density. Figure 2A is plotted on linear axes, and Figure 2D is plotted on double-logarithmic axes. The white cloud of points represents the 22 synthetic density functions, one on top of the other, to depict a range of potential density functions that could arise from the particular $75.9 \%$ power-law mixture. This cloud of simulated density functions captures virtually every point along the curve of the empirical density function, so the empirical density could plausibly be a similar mixture.

For this participant, the proportion of power-law behavior was $\rho_{\mathrm{PL}}=75.9 \%$ of 1,094 simulated pronunciation times. The powerlaw mode was set at $\Omega_{\mathrm{PL}}=6.256 \log$ units $(521 \mathrm{~ms})$, which corresponds to the first hump on the distribution's tail; the inverse power-law exponent $\alpha=5.5$. The parent lognormal had a mode $\Omega_{\mathrm{LN}}=6.256$ in natural logarithm units $(521 \mathrm{~ms})$ and a standard deviation $\sigma=.095 \log$ units $( \pm 50 \mathrm{~ms}$; note that when transformed onto a linear scale, the standard deviation resulting from a given $\sigma$ depends on the value of $\Omega_{\mathrm{LN}}$ and is not symmetric about the mean. We report an average of the linear standard deviations that result from adding and subtracting one $\sigma$ from a given $\Omega_{\mathrm{LN}}$ mean). Of the 1,094 synthetic trials, $24.1 \%$ were drawn exclusively from the fast end of a parent lognormal distribution; all were less than or equal to the lognormal mode. This particular case required no data points from the slow tail (slower than the mean/mode) of the lognormal distribution. The slow tail is apparently exclusively power law. The participant's data were successfully mimicked in a $\rho_{\mathrm{FLN}} 24.1 \%+\rho_{\mathrm{BLN}} 0 \%+\rho_{\mathrm{PL}} 75.9 \%=100 \%$ mix of lognormal and power-law behavior.

Looking more closely at details, this participant's density has a dramatically stretched slow tail, as seen in Figure 2A. On the linear axes of Figure 2A, the slow tail begins its dramatic decline from the mode ( $\approx 521 \mathrm{~ms}$ ) extending, at least, through the $1,100-\mathrm{ms}$ mark. When replotted on double-logarithmic axes in Figure 2D, the previously stretched tail falls off approximately as a line. The slow tail in Figure 2D is apparently an inverse power law spanning an interval of about $600 \mathrm{~ms}$ beyond the distribution's mode, about 2.78 decades of response time. This density illustrates a category of density functions that we call hereafter power-law dominant. The solid black lines in Figures 2A and 2D depict one of only two power-law dominant pronunciation-time distributions present in the 20 participants' data sets.

Intermediate mixtures. Figure 2B illustrates an intermediate mixture of power-law and lognormal distributions. Synthetic density functions are plotted as white points behind the participant's empirical density (and on log-log axes in Figure 2E). The white cloud represents all 22 synthetic samples and again supplies a potential range of density functions that can arise from the particular $\rho_{\mathrm{PL}}=38 \%$ inverse power-law mixture. The synthetic densities capture the participant's density function, so the participant's data could plausibly be a similar mixture.

For this participant, $\rho_{\mathrm{PL}}=38 \%$ of 1,096 data points were drawn in each of 22 simulations from the same power-law distribution. The exponent of the power-law $\alpha=7.5$, and the power-law mode $\Omega_{\mathrm{PL}}=6.253 \log$ units $(520 \mathrm{~ms})$. The remaining $62 \%$ of the samples were taken from a lognormal parent with a mode $\Omega_{\mathrm{LN}}=$ $6.253 \mathrm{log}$ units, or $520 \mathrm{~ms}$, and a standard deviation $\sigma=.095 \mathrm{log}$ units $( \pm 49 \mathrm{~ms})$. Of synthetic and empirical data, $42.8 \%$ are less than or equal to the lognormal mode, which means $19.2 \%$ of data points came from the lognormal tail $\left(\rho_{\mathrm{FLN}} 42.8 \%,+\rho_{\mathrm{BLN}} 19.2 \%\right.$ $+\rho_{\mathrm{PL}} 38 \%=100 \%$ ).

Power-law behavior is much less pronounced in the slow tail of this participant's density, compared with Figure 2D's density. Yet the slow tail in Figure 2B still declines more or less linearly, on log axes, only at much faster rate. The more rapid decay of the stretched slow tail and the more symmetric shape are mimicked with an intermediate mix of power-law and lognormal behavior. So, we call such examples intermediate mixtures. Fourteen participants' data sets were matched with intermediate mixtures.

Lognormal dominant. Figure 2C illustrates another 22 synthetic density functions plotted as white points behind a participant's empirical density. This cloud depicts the potential range of density functions that can arise from the particular $\rho_{\mathrm{PL}}=9 \%$ power-law mixture. Figure $2 \mathrm{~F}$ portrays the empirical and simulated density functions on double-logarithmic axes. As before, the cloud of simulated density functions captures the shape of the empirical function, so the participant's data could be a similar mixture.

For this participant, the sample proportion of power-law behavior was only $\rho_{\mathrm{PL}}=9 \%$ of 1,100 simulated pronunciation times. The power-law mode $\Omega_{\mathrm{PL}}=6.4 \log$ units $(602 \mathrm{~ms})$, and the inverse power-law exponent $\alpha=10$. The parent lognormal had a mode $\Omega_{\mathrm{LN}}=6.304$ in natural logarithm units $(547 \mathrm{~ms})$ and a standard deviation $\sigma=.09 \log$ units $( \pm 49 \mathrm{~ms})$. The remaining $91 \%$ of the 1,100 synthetic trials were drawn from the parent lognormal distribution of which $44.5 \%$ were less than or equal to the lognormal mode, leaving $46.5 \%$ of data points from the lognormal tail $\left(\rho_{\mathrm{FLN}} 44.5 \%+\rho_{\mathrm{BLN}} 46.5 \%+\rho_{\mathrm{PL}} 9 \%=100 \%\right)$.

On double-log axes, the third participant's data resemble a symmetric, downturned parabola depicted by the solid black curve in Figure 2F. This is also how idealized lognormal densities appear on double-logarithmic axes (see Figure 1C). Given the close resemblance, we called this third kind of density lognormal dominant. The solid black lines in Figures $2 \mathrm{C}$ and $2 \mathrm{~F}$ represent one of four lognormal dominant distributions.

\section{Simulations of Participant Hazard Functions}

One simple arithmetic principle captures variation in pronunciation times, namely, multiplicative interaction among random variables. Hypothetical cocktails of power-law and lognormal behavior successfully mimicked the dispersion of each and every participant's data. In each case, repeatedly replicated synthetic distributions established $90 \%$ confidence intervals around the empirical density. These detailed matches between empirical and simulated probability density functions are encouraging, but the hazard function test is more conservative.

Next, we combined the discriminatory power of a hazard function analysis with nonparametric bootstrapping (Efron \& Tibshiriani, 1993). The bootstrap procedure is based on resampling techniques to compute standard errors and conduct statistical tests using empirical distributions with unknown population distributions. We used the bootstrap procedure to transform hazard function simulations into a quantitative statistical test.

\section{Hazard Functions}

What exactly is a hazard function? Hazard functions track the continuously changing likelihood of an event, for instance, that a 
response will occur given that some time has passed and it has not already occurred. The empirical hazard function of response times estimates the probability that a response will occur in a given interval of time, provided that it has not already occurred (Chechile, 2003; Luce, 1986; Van Zandt \& Ratcliff, 1995). It is calculated using the probability density and cumulative density functions of a participant's response times.

Equation 3 is a formal definition in which $t$ refers to the time that has passed without a response on an increasing time axis, $\mathrm{f}(t)$ is the probability density as a function of time, and $\mathrm{F}(t)$ is the cumulative distribution function as a function of time.

$$
\mathrm{h}(t)=\mathrm{f}(t) \div[1-\mathrm{F}(t)] .
$$

The hazard rate is represented graphically by plotting the successive time intervals against their associated probabilities. It is straightforward to compute a hazard function from a histogram, except that the histogram method generally yields unstable hazard function estimates. This is especially true in the slow tail of the distribution where the hazard function tracks a ratio of two numbers that approach zero as they close in on the slow tail's end.

The previous difficulty can never be fully eliminated, but it can be minimized somewhat by using very large data sets and a random smoothing technique described by Miller and Singpurwalla (1980). The latter technique divides the time axis so that each time interval uses equal sample sizes. While other techniques exist, many previous studies have also used this method, and so, our results compare with the existing literature.

Response time hazard functions commonly increase to a peak, decrease, and then level off to a more or less constant value. Peaked hazard functions have interesting and counterintuitive implications. For response times, they mean that when a response does not occur within the time interval up to and including the peak, it becomes less likely to occur at points in the future. This shape is so widely observed that candidate theoretical distributions whose hazard functions cannot mimic this pattern are dismissed out of hand (see discussion in Balakrishnan \& Ashby, 1992; Luce, 1986; but take note also of Van Zandt \& Ratcliff, 1995).

Probability density functions can appear nearly identical, both statistically and to the naked eye, and yet are clearly different on the basis of their hazard functions (but not vice versa). Hazard functions are thus more diagnostic than density functions (Townsend, 1990). On this basis, Luce (1986) rejected many classical and ad hoc models of response time because they lack known qualitative features of empirical hazard functions-no need of more details, such as parameter estimation and density fitting.

Despite their utility, hazard functions remain mostly absent from the wide-ranging response time literature. Perhaps this is because no straightforward inferential statistical test is associated with differences in hazard functions. Instead, hazard functions are usually contrasted qualitatively, in terms of their relative ordering (Townsend, 1990) or with the help of statistical bootstrapping methods.

\section{Characteristic Hazard Functions}

What do hazard functions of power-law and lognormal cocktails look like? They look like the three characteristic hazard functions previously identified by mathematical psychologists. The illustrated hazard functions are plotted on standard linear axes and may be readily compared with hazard functions that appear in the response time literature. Each participant's set of 22 synthetic hazard functions was computed from the same 22 synthetic data sets used to generate the previously described synthetic density functions.

Power-law dominant. Recall that the solid black lines in Figures 2A and 2D each represent an individual participant's empirical, pronunciation-time density function, on linear and doublelogarithmic axes. Plots of 22 separate simulations of the participant's density function are depicted together as clouds of white points. Cocktail simulations generate both probability density and hazard functions simultaneously.

The participant data portrayed in Figure $2 \mathrm{~A}$ is one of two participants' distributions that were power-law dominant. The heavy black curve in Figure $2 \mathrm{G}$ represents the empirical hazard function of the same data, on standard linear axes. In each of the hazard function graphs, the $x$-axis indexes pronunciation time in $\mathrm{ms}$, and the $y$-axis indexes the instantaneous hazard rate for the given interval of pronunciation time.

Hazard functions for a participant's 22 simulations are depicted as white points plotted behind the participant's hazard function, as in Figure 2G. The cloud of points supplies a $90 \%$ confidence interval and a visual sense of the range of hazard function shapes that emerge from repeated simulations using the same mixture of power-law and lognormal behavior.

Notice that the synthetic hazard functions in this example match one class of characteristic hazard functions - hazard functions that rise to an asymptote and stay more or less constant past that point. Hazard functions that level off to constant values imply that the likelihood that a response will occur stays approximately constant into the future. Past a certain point, knowing how much time has elapsed supplies no additional information about the likelihood that a response will be observed. Power-law dominant data also yield this hazard function shape, and 2 of 20 participants' hazard functions fit this description.

Intermediate mixture. Figure $2 \mathrm{H}$ portrays hazard functions for another participant as white points plotted behind the empirical hazard function. The hazard functions of these synthetic densities all rise to a peak and decline to an asymptote past that point. Fourteen of the 20 empirical hazard functions rose to a noticeable peak and then declined to an asymptote. All 14 match the second and most prominent class of hazard functions described by Luce (1986) and Maddox et al. (1998). Intermediate-mixture density functions yield these characteristic peaked hazard functions.

Lognormal dominant. Four of the 20 participants produced hazard functions that rose quickly to a much higher peak and fell off sharply, which matches the third class of characteristic hazard functions. Lognormal dominant simulated data mimicked this class of hazard functions. The solid black line in Figure 2I portrays the hazard function of one participant's data plotted in Figure $2 \mathrm{C}$, and the cloud of hazard functions in Figure 2I again illustrates the range of shapes that emerged from repeated simulations using the same mixture.

\section{Discussion}

The cocktail hypothesis yields a continuum of mixtures that encompass all three distinct hazard function categories. End points at the extreme ends of the continuum are simulations of lognormal 
dominant versus power-law dominant distributions. These extremes are most tightly constrained by the data. Intermediate mixtures are less constrained and likely support multiple parameterizations in some cases. Nonetheless, the intermediate cases do not require much beyond what is gotten from the extreme ends of the continuum. They are parsimonious with these two extremes.

Thus, the account is anchored at the extremes in the choice of lognormal and power-law behaviors, and the intermediate cases follow without additional assumptions. In each case, 22 replications of synthetic distributions establish $90 \%$ confidence intervals around the corresponding hazard function. Thus, multiplicative interaction among random variables again captures participants' dispersion, this time in the hazard functions of pronunciation times. Most important, synthetic mixtures of power-law and lognormal behavior replicate the three generic shapes that Luce (1986) and Maddox et al. (1998) highlighted as generally characteristic of response time behavior.

The three generic hazard shapes are well documented in the response time literature and occur in a wide variety of experimental contexts. On this basis, Maddox et al. (1998) speculated that the similarity of empirical hazard functions across experimental contexts suggests a common origin. We now propose the common origin to be multiplicative interactions. The sufficiency of the present mix of lognormal and inverse power-law behavior supports this proposal.

Notably, the motivation in evidence for multiplicative interactions is more reliable than the motivation for any specific cocktail model. The fixed parameters of each participant's parent distributions were adopted as a simplifying assumption (cf. Van Zandt \& Ratcliff, 1995). Thus, these particular simulations establish that relatively constrained cocktails of multiplicative interactions are sufficient to capture salient empirical details of response time density and hazard functions, the same details that have frustrated previous modeling efforts.

\section{A Second, More Inclusive Existence Proof}

To this point, we have conducted a conservative test of simulated response time densities, using hazard functions, to identify the kind of system dynamics that underlie cognitive response times. To the extent that power-law behavior is diagnostic, system dynamics are interaction dominant dynamics. We next generalize this result to a new data set more broadly inclusive of variation in word-naming performance.

This is one kind of model testing approach. The value of the test is simply that it compares the model's capacity to mimic human performance in data that are more inclusive of the performance at issue (cf. Kirchner, Hooper, Kendall, Neal, \& Leavesley, 1996). This kind of model testing is seen, for instance, when a connectionist model of word naming is tested on a larger or different word population. The second existence proof includes a wider variety of words to further explore participant individual differences in dispersion of pronunciation times.

The second existence proof also includes another test of the cocktail hypothesis in a contrast with ex-Gaussian simulations of response time distributions. Ex-Gaussians resemble power-lawlognormal cocktails in that both convolve a distribution and a skewed slow-tail curve. The ex-Gaussian has been introduced several times in history and is known to closely mimic the details of response time probability density functions (Andrews \& Heathcote, 2001; Balota \& Spieler, 1999; Luce, 1986; Moreno, 2002; Ratcliff, 1979; Schmiedek, Oberauer, Wilhelm, Süß, \& Wittmann, 2007; Schwarz, 2001).

\section{Method}

\section{Participants}

Thirty California State University, Northridge, introductory psychology students participated in exchange for course credit.

\section{Stimuli}

From a 23,454-word corpus described in Stone, Vanhoy, and Van Orden (1997), 1,100 target words were selected at random. They comprised 4- to 15-letter words $(M=6.53, S D=2.07)$, ranging from 2 to 15 phonemes $(M=5.49, S D=2.02)$, from one to five syllables $(M=2.08, S D=1.06)$, and in frequency from 5 to 5,146 per million $(M=70.2, S D=295.16$; Kuçera \& Francis, 1967). By contrast, the 1,100 targets in the initial existence proof were sampled from the more narrowly circumscribed corpus of Spieler and Balota (1997), which were four- to five-letter words $(M=4.45, S D=.5)$, with three to five phonemes $(M=3.59$, $S D=.61)$, all single syllable, ranging in frequency from 1 to 10,601 per million $(M=86.81, S D=458.28$; Kuçera \& Francis, 1967).

\section{Procedure}

A participant was presented with each of the 1,100 target words, one per trial, in a random order. Each trial began with a fixation signal $(+++)$ visible for $172 \mathrm{~ms}$ (12 raster refresh cycles) followed after $200 \mathrm{~ms}$ by the word to be named. Participants were instructed to pronounce the word aloud quickly and accurately into a microphone. Each word appeared in the center of a computer monitor controlled by DMASTR software (Forster \& Forster, 1996) running on a PC.

A word target remained on the screen for $200 \mathrm{~ms}$, after its pronunciation tripped a voice key, but no longer than $972 \mathrm{~ms}$ from presentation. If no response was recorded, trials timed out after $10 \mathrm{~s}$. The voice key was reliable to within $1 \mathrm{~ms}$. The experimenter sat quietly, well behind the participant, and recorded pronunciation errors. Each response was followed by a fixed, 629-ms, intertrial interval. Every participant completed 45 practice trials and then the 1,100 experimental trials, which required about $45 \mathrm{~min}$.

\section{Results}

Four analyses were conducted. The first analysis tested for fractal structure in the form of $1 / \mathrm{f}$ scaling in the pattern of variation of response times across response trials. The second tested whether the cocktail simulations adequately mimicked the distributions of word-pronunciation times, to replicate and extend the first existence proof. The third analysis compared parameters of the cocktail mixtures for the two existence proofs and descriptive parameters of empirical distributions and cocktail mixtures. The fourth analysis contrasted the hazard functions of cocktail mixtures, exGaussian mixtures, and empirical pronunciation times. 


\section{Fractal Scaling}

The first analysis was conducted to test for 1/f scaling in each individual's trial series of pronunciation times, to replicate Van Orden, Holden, and Turvey (2003) and Thornton and Gilden (2005). 1/f scaling in a trial-ordered data series supplies independent converging evidence of multiplicative interactions and interaction dominant dynamics, a point we return to in the discussion (see also Van Orden et al., 2003). The rationale and details behind the procedures are described in Holden (2005).

We again included the small proportion of pronunciation times that resulted in errors because it did not change the outcome $(M=$ $2.45 \%, S D=1.84 \%)$. As a first step, all naming times shorter than $200 \mathrm{~ms}$ and longer than 3,000 ms were eliminated from each series. After that, naming times that fell beyond \pm 3 standard deviations from the series mean were eliminated. More than 1,024 data points remained after the censoring procedure, and the initial observations were truncated so that each series comprised 1,024 observations, with one exception. The exception required a larger 5,000 -ms truncation value to ensure 1,024 observations in the data series.

An initial 511-frequency power spectral density analysis was computed, and the power spectra were examined visually for consistency with the fractional Gaussian noise model. Next, the spectral exponents of the power spectra were computed, using methods described in Holden (2005). Three different statistical analyses were conducted on the 29 remaining series (excluding that of Participant 9, which did not pass the visual test for fractional Gaussian noise). Linear and quadratic trends were removed from the series, and the analyses were limited to scales equal to or below one fourth the size of the series to minimize the impact of the detrending procedure on the analyses (see Holden, 2005; Van Orden, Holden, \& Turvey, 2003, 2005).

The first analysis was a 127 -frequency window-averaged spectral analysis. The mean overall spectral exponent for the 29 series that were straightforwardly consistent with the fractional Gaussian noise description was $.21(S D=.14)$. Twenty-seven of the 30 series displayed larger spectral exponents than those of a randomly shuffled version of the same trial series-otherwise known as a surrogate trial series (Theiler, Eubank, Longtin, Galdrikian, \& Farmer, $1992 ; p<.05$ by a sign test).

The average fractal dimension, computed using the standardized dispersion statistic, was $1.39(S D=.06)$. The fractal dimension for white noise is 1.5 . Twenty-eight of the 30 series yielded smaller fractal dimensions than their shuffled surrogate counterparts $(p<$ .05 by a sign test). This outcome was in close agreement with an average fractal dimension of $1.40(S D=.06)$ produced by the detrended fluctuation analysis (Peng, Havlin, Stanley, \& Goldberger, 1995) in which 29 of the 30 series yielded smaller fractal dimensions than their surrogate counterparts $(p<.05$ by a sign test).

The outcomes of these fractal analyses replicate previous reports of fractal $1 / \mathrm{f}$ scaling in pronunciation-time trial series conducted under similar conditions. The average spectral exponent of the data in the first existence proof, reported in Van Orden, Holden, and Turvey (2003), was .29 ( $S D=.10)$ with an average fractal dimension of $1.40(S D=.06)$. The reliable difference in average spectral exponents between the first and second existence proofs, $t(47)=$ $2.20, p<.05$, suggests that the heterogeneity of the inclusive targets tended, on average, to induce weaker patterns of fractal 1/f scaling. However, the average fractal dimensions were not reliably affected. The discrepancy may be due to the spectral analysis being more readily influenced by changes at the scale of individual trials (Holden, 2005).

As an additional check, we also computed an eight-point power spectrum, averaged across participants, as described in Thornton and Gilden (2005). For this analysis, we expanded the maximum response time to $5 \mathrm{~s}$, and included all 30 series in the analysis. We then used a simplex fitting routine to estimate the parameters of a mixture of $1 / \mathrm{f}$ noise and white noise that could yield the same eight-point power spectrum.

A simple sum of a normalized $1 / \mathrm{f}$ noise $(M=0, S D$ and variance $=1$ ) with a spectral exponent of .61 and a zero-mean white noise with a variance of 1.29 yields a power spectrum very much like the trial series of pronunciation times, $\chi^{2}(7)=.08, p>$ .05 (see Thornton \& Gilden, 2005, for details). The analysis produced almost the same parameters when rerun using only the series that met the visual 511-frequency spectral analysis and passed all three of the above sign tests. Overall, the pronunciation trial series displayed evidence of fractal scaling consistent with the earlier reports. Tables 2 and 3 list the spectral exponents and fractal dimensions for the data series from the two existence proofs.

The contrast of spectral exponents suggests the more inclusive existence proof yielded a weaker pattern of fractal $1 / \mathrm{f}$ scaling. It is possible that relative presence of fractal $1 /$ f scaling in a trial series trades off with the relative dispersion in response time. All other things being equal, wider dispersion of response time may yield more whitened patterns of fractal $1 / \mathrm{f}$ scaling. This finding is noteworthy because no statistically necessary relation exists between degree of dispersion and the presence of scaling behavior in trial series. However, the evidence at this point is merely suggestive, and we address this question more directly in separate studies.

\section{Cocktail Simulations}

We used the statistical procedures described in the first existence proof to estimate initial parameters and simulate the pronunciation-time distributions of each individual participant. As expected, this more inclusive heterogeneous set of words yielded a more heterogeneous set of pronunciation-time distributions compared with naming data in the first existence proof. For the cocktail simulations, overall, the power-law modes and the lognormal means were shifted toward slower values.

Twenty-three of the 30 participants' distributions were successfully approximated using modes from the empirical distribution's mode statistics. For the 20 bimodal participants' data, the average difference between the lognormal mode $\Omega_{\mathrm{LN}}$ and the power-law mode $\Omega_{\mathrm{PL}}$ was $53 \mathrm{~ms}$, an increase of $24 \mathrm{~ms}$ from the average difference for bimodal participants in the first existence proof.

Cocktail mixtures successfully mimicked 29 of 30 density and hazard functions. The single failure came from Participant 13. The front fast half of this participant's density was well approximated by samples from a lognormal density, but a double-logarithmic plot of the density revealed a slow tail that decayed faster than linear. This could mean, for example, that 
Table 2

Spectral Exponents and Fractal Dimension Statistics for the Second Existence Proof

\begin{tabular}{|c|c|c|c|}
\hline Participant & $\begin{array}{c}\text { Spectral } \\
\text { exponent }\end{array}$ & $\begin{array}{c}\text { SDA } \\
\text { fractal } \\
\text { dimension }\end{array}$ & $\begin{array}{l}\text { DFA fracta } \\
\text { dimension }\end{array}$ \\
\hline 1 & .07 & 1.45 & 1.46 \\
\hline 2 & .32 & 1.42 & 1.38 \\
\hline 3 & .07 & 1.42 & 1.43 \\
\hline 4 & .15 & 1.41 & 1.42 \\
\hline 5 & .15 & 1.42 & 1.46 \\
\hline 6 & .43 & 1.33 & 1.35 \\
\hline 7 & .25 & 1.38 & 1.37 \\
\hline 8 & .26 & 1.33 & 1.35 \\
\hline 9 & & & \\
\hline 10 & .15 & 1.36 & 1.40 \\
\hline 11 & .46 & 1.27 & 1.31 \\
\hline 12 & .17 & 1.38 & 1.42 \\
\hline 13 & .23 & 1.42 & 1.45 \\
\hline 14 & -.05 & 1.47 & 1.48 \\
\hline 15 & .16 & 1.44 & 1.39 \\
\hline 16 & .04 & 1.44 & 1.44 \\
\hline 17 & .24 & 1.36 & 1.42 \\
\hline 18 & .14 & 1.43 & 1.42 \\
\hline 19 & .56 & 1.25 & 1.22 \\
\hline 20 & .11 & 1.46 & 1.49 \\
\hline 21 & .10 & 1.40 & 1.42 \\
\hline 22 & .39 & 1.35 & 1.34 \\
\hline 23 & .32 & 1.37 & 1.35 \\
\hline 24 & .42 & 1.27 & 1.25 \\
\hline 25 & .09 & 1.42 & 1.42 \\
\hline 26 & .32 & 1.36 & 1.40 \\
\hline 27 & .06 & 1.48 & 1.43 \\
\hline 28 & .33 & 1.39 & 1.42 \\
\hline 29 & .11 & 1.38 & 1.41 \\
\hline 30 & .19 & 1.34 & 1.38 \\
\hline$M$ & .21 & 1.39 & 1.40 \\
\hline$S D$ & .14 & 0.06 & 0.06 \\
\hline
\end{tabular}

Note. Spectral exponent and fractal dimension statistics that characterize the fractal scaling in the naming series are depicted for both analyses. The details of the SDA fractal dimension statistic are described in Holden (2005); the details of the DFA fractal dimension statistic are described in Peng, Havlin, Stanley, and Goldberger (1995). Participant 1's series contained many extreme observations, and a more liberal 5-s cutoff was required to recover at least 1,024 observations for the fractal analyses. Participant 9 was eliminated from the analyses because visual inspection of the 512-frequency spectral plot revealed an inverted $U$-shaped spectrum. $\mathrm{SDA}=$ standardized dispersion analysis; DFA $=$ detrended fluctuation analysis.

slow times are consistent with an exponential tail instead of a power-law tail.

However, the tail also contained multiple extreme observations that deviated from the otherwise exponential pattern, more in line with power-law behavior. Thus, for instance, the data could reflect a power law, partially collapsed into a lognormal curve, as that pattern could also appear like the data from Participant 13. Nonetheless, we counted this as a failure to disambiguate, one way or the other. Table 4 lists the parameter values of the parent distributions for each participant's simulated data.

Power-law dominant. Three of 30 empirical density functions displayed hazard functions consistent with power-law dominant behavior. Exponents of the parent power-law distributions ranged from 3 to 3.25 , and the $\rho_{\mathrm{PL}}$ mixture proportions ranged from $65 \%$ to $47 \%$. Lognormal means ranged from 6.24 to 6.37 on a log scale, or 513 to $584 \mathrm{~ms}$, with lognormal standard deviations set between .105 and $.135 \log$ units. These densities were best approximated by allowing the mode of the parent power-law distribution to fall slightly beyond the mode of the parent lognormal distribution, by .01 to $.15 \log$ units.

Figure $3 \mathrm{~A}$ depicts the power-law dominant distribution of one participant. The sample proportion of power-law behavior was $\rho_{\mathrm{PL}}=65 \%$ of 1,029 simulated pronunciation times. The powerlaw mode was set at $\Omega_{\mathrm{PL}}=6.41 \log$ units, or $608 \mathrm{~ms}$, and the inverse power-law exponent was $\alpha=3$. The remaining 35\% of the 1,029 synthetic trials were drawn from a parent lognormal distribution with a mode of $\Omega_{\mathrm{LN}}=6.26$, or $523 \mathrm{~ms}$, and a standard deviation $\sigma=.1 \log$ units, or $53 \mathrm{~ms}$. Of this $35 \%$ of synthetic times, $16 \%$ were less than or equal to the lognormal mode, and $19 \%$ exceeded the lognormal mode $\left(\rho_{\mathrm{FLN}} 16 \%+\rho_{\mathrm{BLN}} 19 \%+\rho_{\mathrm{PL}}\right.$ $65 \%=100 \%$ ).

Intermediate mixture. Twenty-three of the 30 empirical hazard functions were simulated with intermediate mixtures of lognormal and inverse power-law behavior. Power-law exponents ranged from 4.5 to 9 , and the $\rho_{\mathrm{PL}}$ mixture proportions ranged from $68 \%$ to $8 \%$. Lognormal means ranged from 6.13 to $6.65 \log \mathrm{ms}$, with standard deviations that ranged from .085 to $.135 \mathrm{log}$ units. Fourteen of the 23 densities were best approximated if the mode of the parent power-law distribution was allowed to fall slightly beyond the mode of the parent lognormal by .008 to $.16 \log$ units.

Figure 3B illustrates the intermediate mix of one participant: $\rho_{\mathrm{PL}}=40 \%$ of the 1,097 data points drawn in each of 22 simula-

Table 3

Spectral Exponents and Fractal Dimension Statistics for the First Existence Proof

\begin{tabular}{cccc}
\hline Participant & $\begin{array}{c}\text { Spectral } \\
\text { exponent }\end{array}$ & $\begin{array}{c}\text { SDA fractal } \\
\text { dimension }\end{array}$ & $\begin{array}{c}\text { DFA fractal } \\
\text { dimension }\end{array}$ \\
\hline 1 & .40 & 1.34 & 1.31 \\
2 & .21 & 1.41 & 1.46 \\
3 & .28 & 1.44 & 1.47 \\
4 & .25 & 1.42 & 1.42 \\
5 & .14 & 1.43 & 1.45 \\
6 & .22 & 1.51 & 1.47 \\
7 & .40 & 1.33 & 1.31 \\
8 & .28 & 1.35 & 1.39 \\
9 & .41 & 1.41 & 1.37 \\
10 & .35 & 1.35 & 1.33 \\
11 & .20 & 1.52 & 1.50 \\
12 & .49 & 1.30 & 1.32 \\
13 & .38 & 1.33 & 1.41 \\
14 & .19 & 1.40 & 1.41 \\
15 & .27 & 1.40 & 1.45 \\
16 & .22 & 1.45 & 1.31 \\
17 & .40 & 1.33 & 1.47 \\
18 & .16 & 1.47 & 1.40 \\
19 & .22 & 1.37 & 1.41 \\
20 & .29 & 1.40 & 1.40 \\
$M$ & .29 & 1.40 & 0.07 \\
$S D$ & .10 & 0.06 & \\
\hline & & & \\
\hline
\end{tabular}

Note. Participants were numbered according to the value of the scaling exponent that characterized the decay in the slow tail of their response time distribution. This table supplies a different ordering of the same spectral and fractal dimension statistics that appeared in Van Orden, Holden, and Turvey $(2003$, p. 342). SDA = standardized dispersion analysis; DFA = detrended fluctuation analysis. 
Table 4

Parameters Used to Generate Synthetic Distributions in the Second Existence Proof

\begin{tabular}{|c|c|c|c|c|c|c|c|}
\hline Participant & $\Omega_{\mathrm{LN}}$ & $\sigma$ & $\Omega_{\mathrm{PL}}$ & $\alpha$ & $\rho_{\mathrm{FLN}}$ & $\rho_{\mathrm{BLN}}$ & $\rho_{\mathrm{PL}}$ \\
\hline 1 & 6.260 & .100 & 6.410 & 3.00 & .160 & .190 & .650 \\
\hline 2 & 6.370 & .115 & 6.480 & 3.25 & .227 & .213 & .560 \\
\hline 3 & 6.337 & .135 & 6.350 & 3.00 & .339 & .191 & .470 \\
\hline 4 & 6.312 & .135 & 6.312 & 4.50 & .377 & .143 & .480 \\
\hline 5 & 6.244 & .100 & 6.244 & 5.00 & .317 & .003 & .680 \\
\hline 6 & 6.373 & .110 & 6.480 & 4.74 & .259 & .311 & .430 \\
\hline 7 & 6.506 & .110 & 6.620 & 5.00 & .274 & .346 & .380 \\
\hline 8 & 6.423 & .120 & 6.590 & 6.50 & .313 & .367 & .320 \\
\hline 9 & 6.292 & .135 & 6.440 & 6.00 & .445 & .325 & .230 \\
\hline 10 & 6.123 & .090 & 6.130 & 6.50 & .315 & .001 & .684 \\
\hline 11 & 6.123 & .100 & 6.130 & 7.25 & .398 & .001 & .601 \\
\hline 12 & 6.339 & .110 & 6.350 & 7.25 & .436 & .005 & .560 \\
\hline 13 & 6.378 & .100 & 6.378 & 7.25 & .433 & .077 & .490 \\
\hline 14 & 6.372 & .115 & 6.380 & 6.25 & .375 & .000 & .625 \\
\hline 15 & 6.377 & .090 & 6.500 & 7.25 & .310 & .400 & .290 \\
\hline 16 & 6.372 & .090 & 6.372 & 6.50 & .372 & .001 & .627 \\
\hline 17 & 6.208 & .110 & 6.208 & 8.25 & .454 & .000 & .546 \\
\hline 18 & 6.284 & .110 & 6.284 & 6.00 & .452 & .148 & .400 \\
\hline 19 & 6.196 & .110 & 6.310 & 8.00 & .370 & .380 & .250 \\
\hline 20 & 6.330 & .130 & 6.490 & 8.75 & .518 & .402 & .080 \\
\hline 21 & 6.248 & .120 & 6.248 & 5.00 & .482 & .218 & .300 \\
\hline 22 & 6.303 & .120 & 6.303 & 6.00 & .484 & .266 & .250 \\
\hline 23 & 6.159 & .100 & 6.280 & 9.00 & .315 & .425 & .260 \\
\hline 24 & 6.414 & .130 & 6.414 & 9.00 & .507 & .000 & .493 \\
\hline 25 & 6.130 & .085 & 6.230 & 8.50 & .226 & .284 & .490 \\
\hline 26 & 6.653 & .125 & 6.740 & 7.00 & .438 & .312 & .250 \\
\hline 27 & 6.232 & .110 & 6.232 & 8.00 & .453 & .147 & .400 \\
\hline 28 & 6.160 & .115 & 6.210 & 8.75 & .428 & .442 & .130 \\
\hline 29 & 6.436 & .130 & 6.550 & 8.00 & .388 & .492 & .120 \\
\hline 30 & 6.176 & .110 & 6.230 & 9.25 & .383 & .467 & .150 \\
\hline$M$ & 6.304 & .112 & 6.363 & 6.62 & .375 & .219 & .407 \\
\hline
\end{tabular}

Note. This table lists cocktail parameters that approximate each of the 30 empirical density functions of the second existence proof. The participant numbers were established by ordering each distribution in terms of the empirically estimated slope of the tail of the distribution. This explains why the participant numbers approximate a rank ordering of the alpha parameter. Participants 1, 2, and 3 were classified as power-law dominant; Participants 28, 29, and 30 were classified as lognormal dominant. The parameters reported for Participant 13 represent a failure of the cocktail mixture. The remaining participants were classified as intermediate mixtures. Plots of all the empirical and cocktail distributions and hazard functions can be viewed online at http://www.csun.edu/ jgh62212/RTD. $\Omega_{\mathrm{LN}}=$ lognormal mode; $\sigma=$ lognormal standard deviation; $\Omega_{\mathrm{PL}}=$ power-law mode; $\alpha=$ power-law tail; $\rho_{\mathrm{FLN}}=$ proportion in front end of lognormal; $\rho_{\mathrm{BLN}}=$ proportion in back end of lognormal; $\rho_{\mathrm{PL}}=$ proportion power law.

tions from an inverse power-law distribution, the exponent of the power-law $\alpha=6$, and the power-law mode $\Omega_{\mathrm{PL}}=6.284 \log$ units $(536 \mathrm{~ms})$. The remaining $60 \%$ of synthetic data were taken from a lognormal parent with a mode $\Omega_{\mathrm{LN}}=6.284 \log$ units, or $536 \mathrm{~ms}$, and a standard deviation $\sigma=.11 \log$ units, or about $59 \mathrm{~ms}$. Of the remaining $60 \%$ of the synthetic samples, $45.2 \%$ were less than or equal to the mode, and $14.8 \%$ exceeded the lognormal mode $\left(\rho_{\text {FLN }}\right.$ $\left.45.2 \%+\rho_{\mathrm{BLN}} 14.8 \%+\rho_{\mathrm{PL}} 40 \%=100 \%\right)$.

Lognormal dominant. The remaining three empirical densities were lognormal dominant with less power-law behavior sampled from power laws entailing larger exponents. Power-law exponents ranged from 8 to 9.25 , and lognormal mean parameters ranged from 6.16 to 6.463 , with lognormal standard deviations of .1 to .13 . Modes of power-law distributions fell just past modes of lognormals by .05 to $.11 \log$ units.

Figure $3 \mathrm{C}$ illustrates the lognormal dominant mix of one participant: $\rho_{\mathrm{PL}}=15 \%$ of the 1,098 data points drawn in each of 22 simulations, the exponent of the power law was $\alpha=9.25$, and the power-law mode was $\Omega_{\mathrm{PL}}=6.23 \mathrm{log}$ units. The remaining $85 \%$ of synthetic data came from a lognormal parent with a mode $\Omega_{\mathrm{LN}}=$ $6.176 \log$ units, or $481 \mathrm{~ms}$, and a standard deviation $\sigma=.11 \log$ units, or about $53 \mathrm{~ms} ; 38.3 \%$ of synthetic and empirical data were less than or equal to the lognormal mode, leaving $46.7 \%$ of data points from the lognormal tail $\left(\rho_{\mathrm{FLN}} 38.3 \%+\rho_{\mathrm{BLN}} 46.7 \%+\rho_{\mathrm{PL}}\right.$ $15 \%=100 \%)$.

\section{Parameter Contrasts}

The two existence proofs used two different samples of word targets, the principal difference being longer and multisyllabic words in the present sample. With this in mind, we examined distributions of cocktail parameters from both sets of simulations. Figure 4 depicts seven box plots to contrast the parameters required to mimic the two data sets.

The proportion of lognormal data in the front end of simulated distributions stayed most consistent across the two data sets. The inclusive naming study yielded a more heterogeneous distribution of lognormal modes $\left(\Omega_{\mathrm{LN}}\right)$, however, focused on somewhat slower times. This same basic pattern held for the lognormal standard deviations $(\sigma)$ and the power-law mode $\left(\Omega_{\mathrm{PL}}\right)$. Simulations of the inclusive word data tended to exchange samples from the back of the lognormal for more power-law samples and often required 

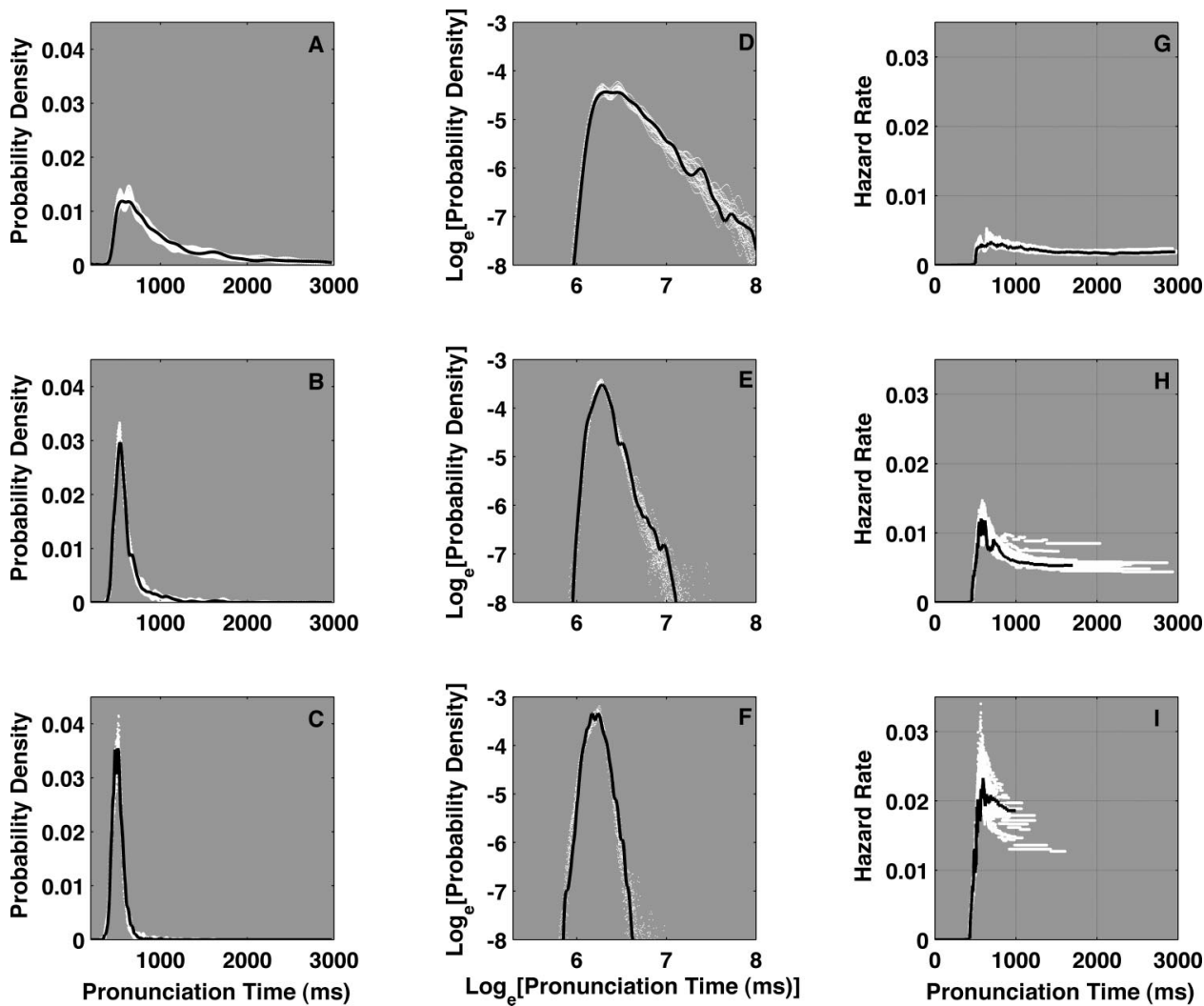

Figure 3. Three individuals' pronunciation-time density functions from the second existence proof are depicted in the same format as Figure 2. Notice that the $x$-axes now extend out to $3 \mathrm{~s}$, twice what is required for the distributions in the first existence proof. Panels A, B, and C depict the kernel-smoothed density functions on linear axes; the solid black line represents the empirical density, and the white cloud of points plotted behind the participant's empirical density represents the density functions of the corresponding 22 cocktail simulations. Panels D, E, and F depict the same densities as in Panels A, B, and C, now on double-logarithmic axes. Panels $\mathrm{G}, \mathrm{H}$, and I depict the empirical hazard functions in black and the 22 simulated hazard functions in white. The boundaries established by the 22 simulated distributions circumscribe $90 \%$ confidence intervals around each empirical probability density and hazard function.

shallower power-law tails (i.e., smaller $\alpha$ ). Other things being equal, these differences imply increases in the dispersion of distributions associated with the inclusive targets, which underscores the importance of accounting for changes in dispersion in theoretical descriptions of word naming and other cognitive tasks (e.g., Andrews \& Heathcote, 2001; Balota et al., 2004; Balota \& Spieler, 1999; Heathcote, Popiel, \& Mewhort, 1991; Schmiedek et al., 2007; Wagenmakers \& Brown, 2007; Yap, Balota, Tse, \& Besner, 2008).

The scatterplots in Figure 5 illustrate how well the cocktail simulations recovered summary statistics of pronunciation-time distributions, means, standard deviations, and the positive pattern of skew. Summary statistics would not likely distinguish the cocktail mixtures from empirical data. Cocktail mixtures also captured the linear correlation between means and standard deviations, which has long been recognized in response time data (e.g., Luce, 1986, pp. 64-66).

In the most recent example, Wagenmakers and Brown (2007) examined the relation between a participant's mean response time and standard deviation and found a clear pattern of linear correlation across a wide range of decision-based response time tasks. They speculated that the relationship is a lawlike property of response time distributions. We conducted a parallel analysis.

The Wagenmakers and Brown (2007) analysis indexed condition means and standard deviations on a participant-byparticipant basis. This analysis held participant constant to examine how different experimental conditions varied with relative task difficulty. By contrast, our analysis holds the task constant to examine individual differences among participants. Thus, whereas Wagenmakers and Brown found that more difficult, slower conditions give more widely dispersed response times, we find that slower participants tend to produce wider dispersion.

Faust, Balota, Spieler, and Ferraro (1999) anticipated both previous outcomes in formal analyses of their rate-amount model, and they reported similar corroboration in data from Hale, Myerson, Faust, and Fristoe (1995). Individual participants' mean response times were positively correlated with their respective standard deviations, and condition means were 

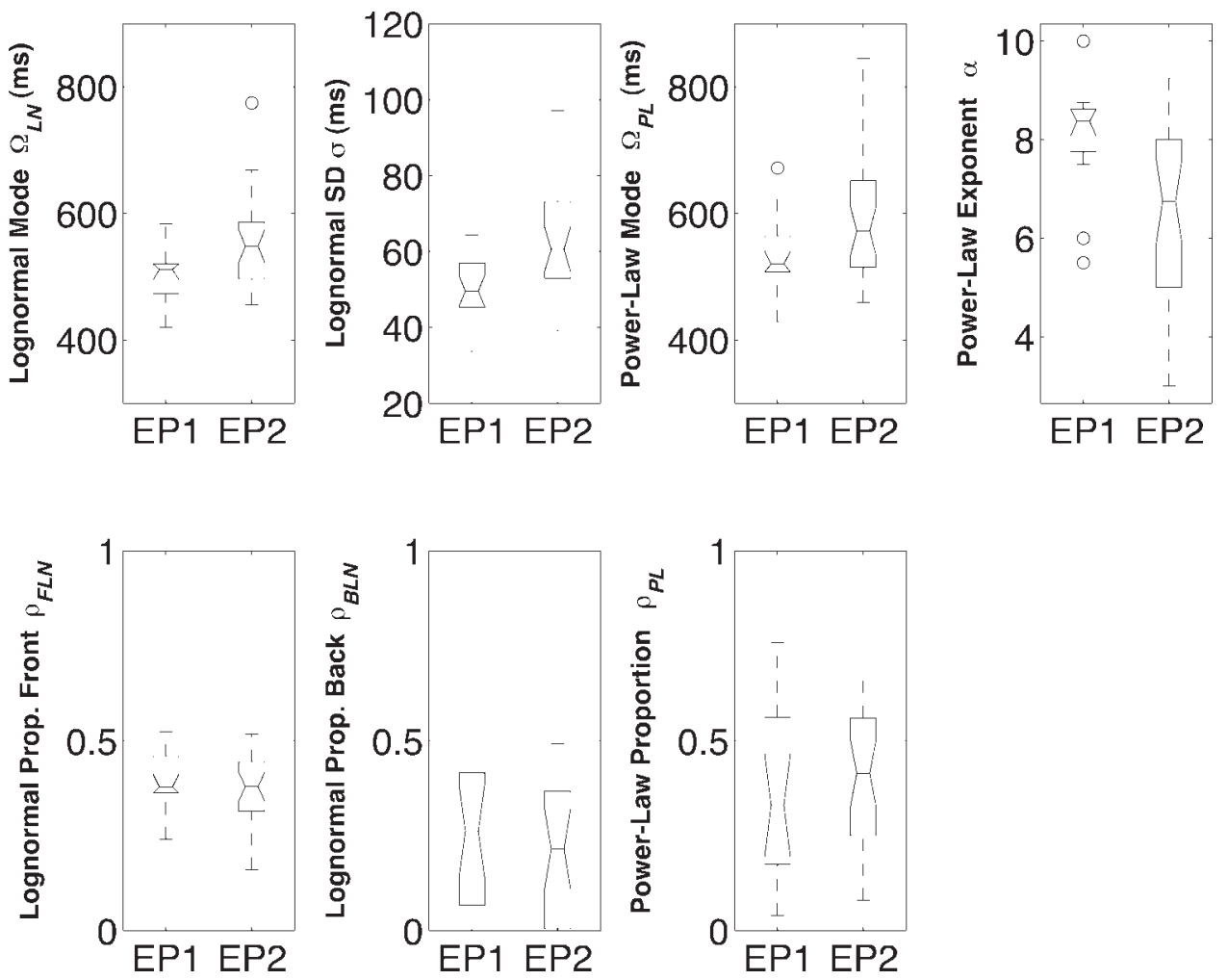

Figure 4. Individual box plots contrast each parameter used to generate the cocktail mixture distributions between the two existence proofs. The EP1 label corresponds to data from the first existence proof, and the EP2 label indicates the second existence proof. For these plots, the lognormal parameters and the power-law modes were converted to linear units of pronunciation time. Distributions of lognormal parameters and power-law modes from EP1 were somewhat faster and less variable than those of the more inclusive EP2. The proportion of lognormal behavior in the fast front ends of cocktail simulations was similar between EP1 and EP2, but the EP2 data sets tended to require more power-law behavior in the slow tails. Prop. = proportion.

positively correlated with standard deviations in the respective condition.

Both existence proofs also yielded reliable correlations between the mean and standard deviation, $r(47)=.86, p<.05$. A scatterplot of the data is presented in the upper left panel of Figure 6. Since word naming is not an explicit decision process, the correlation suggests a more general relationship than Wagenmakers and Brown's (2007) speculation (that it applies primarily to decisionbased tasks). An identical analysis on the synthetic cocktail data yielded a nearly identical relationship between means and standard deviations, $r(47)=.84, p<.05$. A scatterplot of the relationship for the cocktail simulations is presented in the bottom left panel of Figure 6.

In addition, synthetic and empirical scatterplots display a similar pattern of fanning. In the parlance of linear regression, this trapezoidal fanning indicates heterogeneity of error variance. Thus, in addition to displaying the three characteristic hazard functions of response time, the cocktail mixtures also successfully replicate and extend the pervasive pattern of correlation between the mean and standard deviation in response time distributions (Andrews \& Heathcote, 2001; Balota et al., 2004; Balota \& Spieler, 1999; Faust et al., 1999; Heathcote et al., 1991; Holden, 2002; Van Orden, Moreno, \& Holden, 2003; Schmiedek et al., 2007).
Additional relationships are seen across the mean, standard deviation, and skew statistics. A reliable positive correlation exists between participants' mean pronunciation time and a nonparametric estimate of skew, $r(47)=.57, p<.05$, and also between the standard deviation and skew, $r(47)=.76, p<.05$. The same correlations were present in the cocktail mixtures, mean and skew: $r(47)=.60, p<.05 ;$ standard deviation and skew: $r(47)=.79$, $p<.05$. Notably, the positive skew of pronunciation-time distributions implies that the mean, standard deviation, and skew will not generally pass as statistically independent (a point made previously by Van Zandt, 2002).

\section{Ex-Gaussian Contrast}

Finally, we provide additional context for the generality of the cocktail simulations in a contrast with synthetic ex-Gaussian pronunciation-time distributions. A mixture of samples from exponential and Gaussian distributions has long been known to closely approximate empirical response time distributions (e.g., Luce, 1986; Ratcliff, 1979). The word-recognition literature includes the ex-Gaussian as a tool for describing the loci of manipulation effects of factors, such as word frequency, consistency, and even task, that influence the shape of response time distributions 

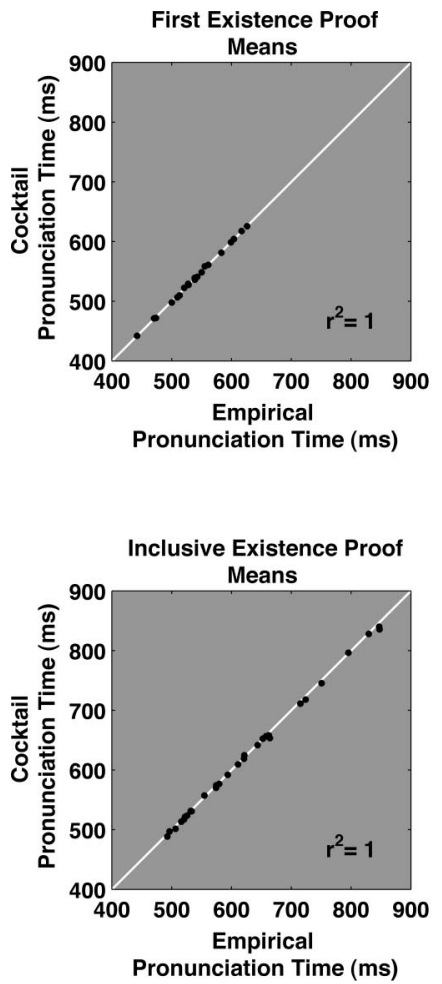
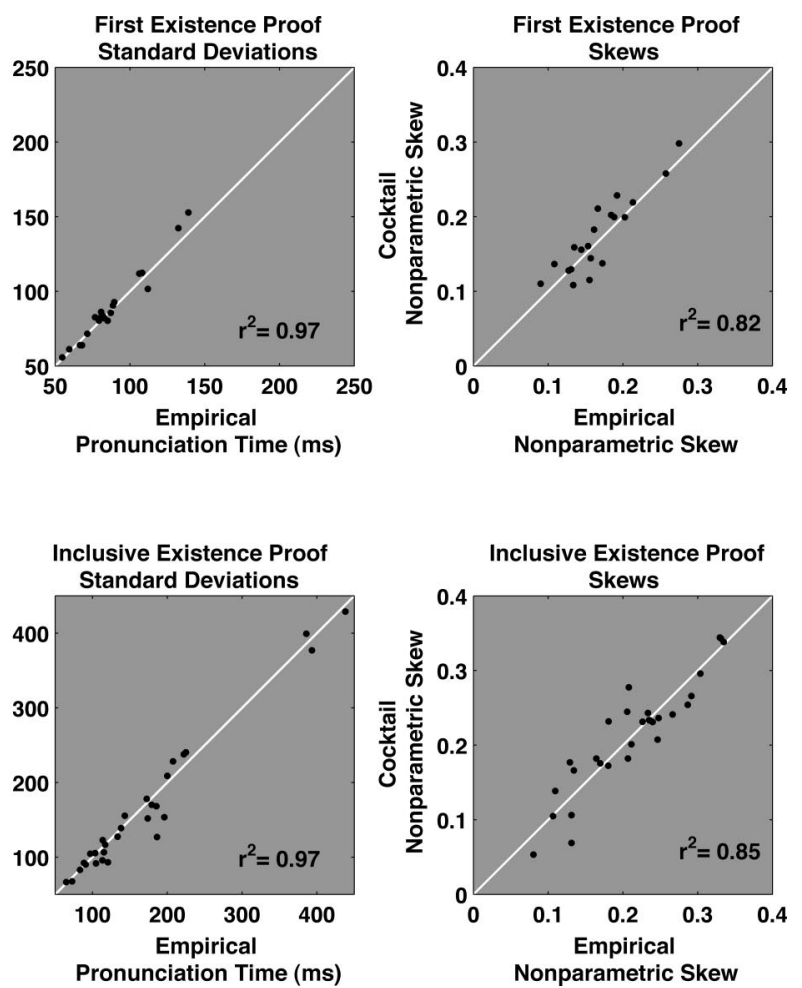

Figure 5. The top three scatterplots compare the pronunciation times from the first existence proof with simulated times in three descriptive statistics: the mean, the standard deviation, and a nonparametric measure of skew $([M-M d] / S D)$. The bottom three panels illustrate the same comparison for the second existence proof (Participant 13 was excluded). The statistics for the simulated times were computed using an omnibus distribution that included all 22 cocktail mixtures. The successful cocktail mixtures reliably recovered empirical means, standard deviations, and skews.

(Andrews \& Heathcote, 2001; Balota et al., 2004; Balota \& Spieler, 1999; Yap et al., 2008).

We used the ex-Gaussian density function to perform maximum-likelihood fits to our pronunciation-time distributions (Van Zandt, 2000). The ex-Gaussian description supplied reasonable fits to the empirical pronunciation-time distributions despite our special effort to collect many more observations than a typical single-session pronunciation experiment. This was true even when contrasted with a simplified three-parameter version of the cocktail that rendered it more comparable to the ex-Gaussian. The simple cocktail convolved a lognormal and inverse power-law distribution using fixed $50 \%-50 \%$ proportions and fixed the power-law mode to the value of the lognormal mean. Only the three remaining parameters, the lognormal mean, standard deviation, and powerlaw exponent, were free to vary in our subsequent nonlinear least squares fits. The ex-Gaussian and both versions of the cocktail mixtures were statistically indistinguishable at the level of the probability density.

As others have pointed out, model selection is not easy to decide on the basis of best fits to probability density functions. It is a different matter to fit both the probability density and the hazard function, however, and the following procedures allowed a contrast of hazard functions for the two kinds of synthetic hazard functions. In addition to the 22 cocktail mixture simulations described earlier, we generated 22 synthetic ex-Gaussian distribu- tions for each participant's empirical distribution. Ex-Gaussian distributions were generated using samples from a synthetic Gaussian and exponential distribution, generated according to the parameters returned by the maximum-likelihood parameter estimation routine.

Both sets of 22 synthetic distributions were collapsed into two respective omnibus distributions, comprising a maximum of 24,200 data points (less if there were fewer than 1,100 observations in the empirical distribution). Following that, we computed hazard functions for each omnibus cocktail and ex-Gaussian synthetic distribution.

Figure 7B depicts the three empirical power-law dominant hazard functions, from the inclusive word data, as three black lines. To their left, depicted as white lines in Figure 7A, are the hazard functions of the omnibus cocktail mixtures that correspond to each empirical distribution depicted in Figure 7B. The synthetic exGaussian omnibus hazard functions for the same three empirical distributions appear as white lines in Figure 7C.

These three plots clarify the power of the hazard function test. The ex-Gaussian hazard functions are qualitatively different from both the empirical and cocktail hazard functions, for example. However, the empirical and cocktail hazard functions share many details in their shapes; they both include subtle peaks and both decay slowly to asymptotic values. By contrast, ex-Gaussian hazard functions simply rise to a constant asymptote. 

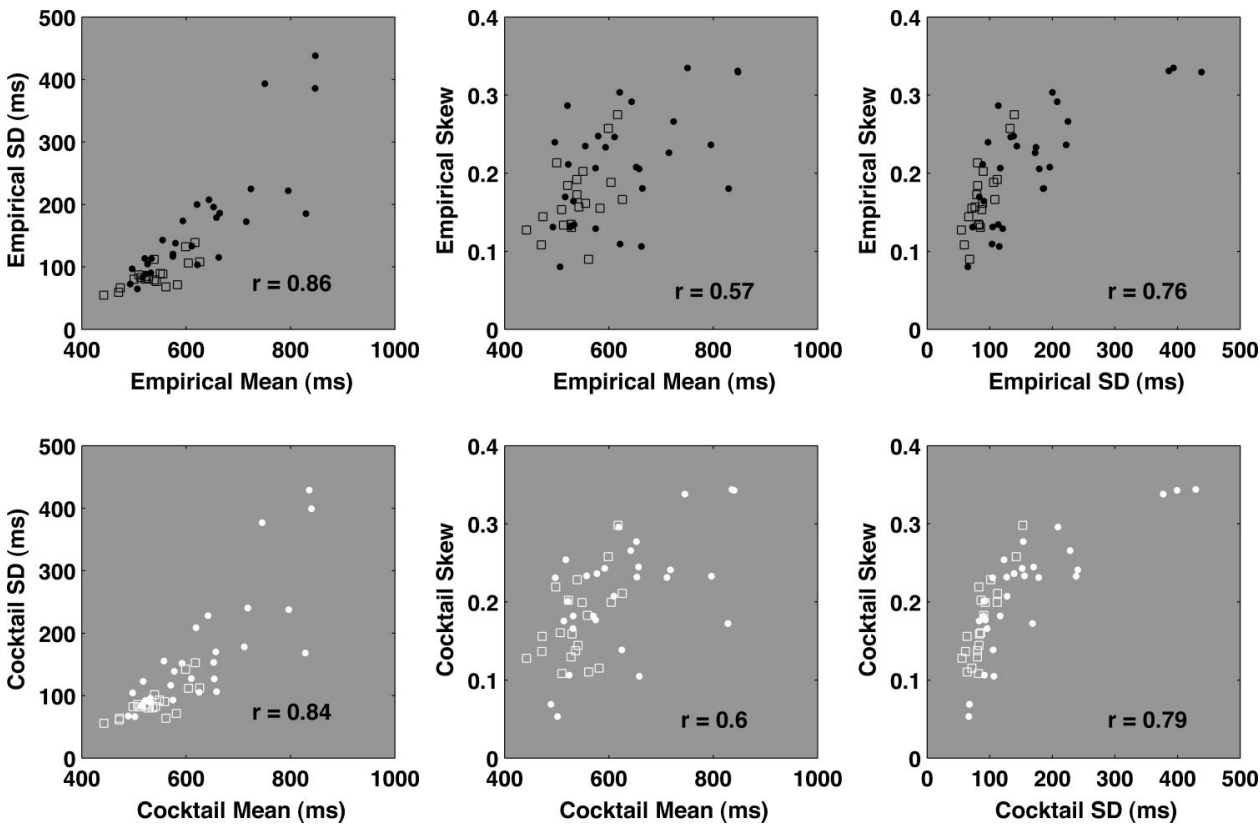

Figure 6. The top three scatterplots depict correlations between mean and standard deviation, mean and skew, and standard deviation and skew for the empirical pronunciation-time distributions. The open squares represent participants from the first existence proof; the filled circles indicate participants from the second existence proof. Since they were an ambiguous case, Participant 13's data were excluded from this and all subsequent analyses. The white markers on the bottom three plots depict the same information for the cocktail simulation of each participant's pronunciation-time distribution. In addition to capturing a comparable magnitude of linear association between the variables, the cocktail mixtures capture additional details of the relationships. For example, both empirical and cocktail mean-standard deviation plots display a fanning pattern as a function of increases in both variables, and both empirical and cocktail standard deviation-skew plots display evidence of a curvilinear relationship.

Figures $7 \mathrm{D}, 7 \mathrm{E}$, and $7 \mathrm{~F}$ are organized to depict the same triptych of contrasts for three lognormal dominant distributions from the inclusive naming task. The two empirical hazard functions with the highest peaks rise to a prominent peak and fall off sharply past that point. The cocktail mixtures in Figure 7D do a much better job of recovering this structure than the ex-Gaussian hazard functions in Figure $7 \mathrm{~F}$.

The third empirical hazard function, with the lowest peak, presents an ambiguous case. It possesses a peak, but the peak is broader than those of the cocktail mixtures. The ex-Gaussian description, on the other hand, does not seem quite peaked enough to capture its shape. This case illustrates that it is largely the properties of the slow tails of distributions that are decisive in discriminating the two frameworks. Lognormal dominant participants tend to produce tighter pronunciation-time distributions with few extreme values, and their hazard functions do not span very large time intervals.

Figure 8 depicts the same triptych of contrasts for distributions comprising intermediate mixes of lognormal and power-law samples. Each panel in the center column of the figure depicts four empirical hazard functions from the inclusive naming study. The third row contains only 3 participants' hazard functions because Participant 13 was excluded as an ambiguous case. The left column of panels depicts the corresponding omnibus cocktail hazard functions; the right column depicts the corresponding omnibus
ex-Gaussian hazard functions. In this figure, one sees most clearly the failure of the ex-Gaussian description. It simply fails altogether to display peaks prominent in all the empirical hazard functions.

We have only reported simulation details of the ex-Gaussian contrast, but we also conducted extracurricular simulations of inverse-Gaussian, ex-Wald, and Weibull alternatives. In contrast to ex-Gaussian and cocktail simulations, the latter distributions all failed Kolmogorov-Smirnov tests and failed to mimic the hazard functions of data from both existence proofs.

We evaluated hazard functions by generating synthetic distributions based on maximum-likelihood fits of the three alternative distributions. The resulting parameter sets supplied each distribution's best approximation to the empirical distributions. We did this mainly because, given certain parameter sets, the inverse Gaussian and exWald can display peaked hazard functions. In fits of the present data, however, the inverse-Gaussian hazard functions generally failed to display peaks. Simulated hazard functions tended to rise too quickly, almost always overtaking the empirical hazard functions.

Weibull hazard functions usually rose monotonically, and too slowly, always undershooting the empirical hazard functions. Only the ex-Wald hazard functions fell in the neighborhood of the empirical hazard functions. Ex-Wald hazard functions rarely exhibited peaks, however. They were qualitatively similar to ex-Gaussian hazard functions but typically failed to track the empirical hazard function as well as the ex-Gaussian. The results of the extracurricular 

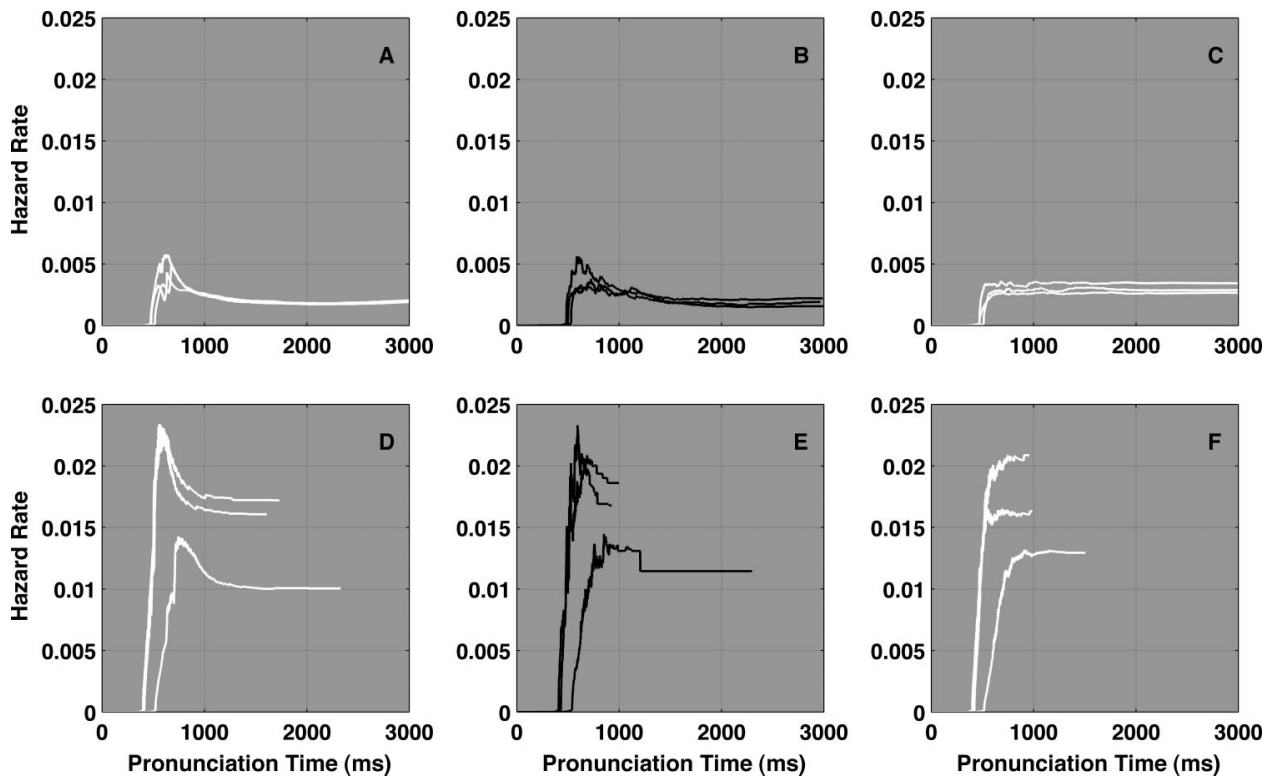

Figure 7. The center column (Panels B and E) depicts apparently power-law dominant and lognormal dominant empirical hazard functions from the second existence proof. Each panel displays three different participants' hazard functions as three black lines. In the left-most column, Panels A and D depict the omnibus hazard functions as three white lines for cocktail mixtures corresponding to empirical Panels B and E. Similarly, in the right-most column, Panels $\mathrm{C}$ and F depict hazard functions of omnibus ex-Gaussian distributions corresponding to the empirical data sets in Panels B and E. The figure makes clear that the shapes of cocktail hazard functions are more consistent with the empirical hazard functions than ex-Gaussian hazard functions.

simulations are not surprising, given that fits to the empirical density functions of the three alternative distributions were generally much poorer than those of the ex-Gaussian.

All these simulations, extracurricular and ex-Gaussian, speak to an obvious worry about whether cocktail mixtures might be too powerful. If power-law-lognormal cocktails can fit any distribution, then successfully mimicking pronunciation-time distributions is expected. Recall that ex-Gaussian simulations are essentially indistinguishable from the empirical distributions and also from the cocktail mixtures, at least for probability density functions. This is why the ex-Gaussian is so popular. Once the hazard functions are examined, however, it is clear that the cocktail descriptions have subtle properties that successfully mimic the empirical hazard functions. Nonetheless, and this is the crux, those same properties render cocktail simulations unable to mimic ex-Gaussian hazard functions.

Figures 7 and 8 demonstrate that cocktail mixture hazard functions are usually poor descriptions of ex-Gaussian hazard functions. Thus, the cocktail mixture scheme cannot fit every possible distribution or hazard function. Taking into account the extracurricular simulations, power-law-lognormal cocktails fail to mimic best-fit density and hazard functions of three common modeldistributions of response time, each tuned to the same empirical distributions as the cocktail mixtures.

Taken together, these four alternate distributions supply perhaps the most plausible empirical forms that a participant's response time distribution could take, other than the cocktail scheme. They are all routinely used to model response time distributions. Nevertheless, the cocktail mixtures fail to represent them reliably. Thus, while the cocktail mixtures are flexible, they are also poised for falsification.
Plausible data patterns exist that are very similar to empirical response times that cocktail simulations fail to accommodate.

The most plausible of these is the ex-Gaussian. Repeatedly in the history of response time research, scientists have either embraced the ex-Gaussian description or pointed out its weaknesses. The ambivalence comes from the excellent fits to response time distributions versus the discrepancy between the analytic form of the ex-Gaussian hazard function and empirical hazard functions (e.g., Ashby et al., 1993; Luce, 1986; Moreno, 2002; Van Zandt 2003). To the discrepancy, we add that ex-Gaussian hazard functions tend to be too short and that they contain too few synthetic data points in their slow tails, a failing that is more prominent in a contrast between exponential and power-law decay.

In conclusion, previous lines of argument that question the ex-Gaussian have been largely analytic in nature, derived from knowledge of the asymptotic behavior of idealized hazard functions. What has been missing is the promise of a motivated alternative that can pass the hazard function test. Interaction dominant dynamics predict multiplicative interactions among component processes and power-law behavior in the slow tails of response time distributions. Sufficiently constrained interaction dominant dynamics also predict lognormal behavior, as we have described. Cocktail combinations of power-law and lognormal behavior pass the hazard function test.

\section{Discussion}

Employing a more inclusive and representative sample of the English language revealed a more heterogeneous collection of 

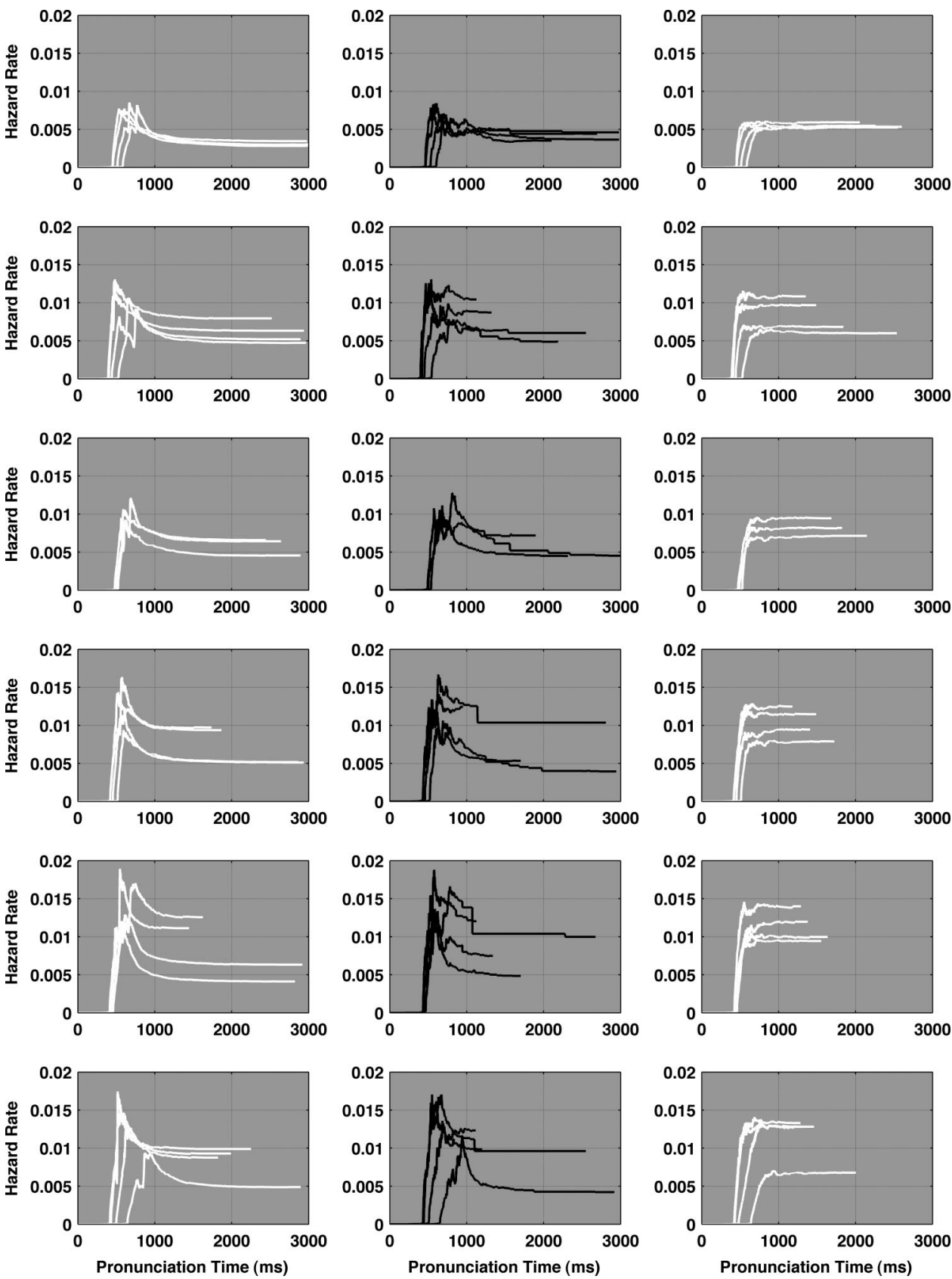

Figure 8. The center column of panels depicts the apparently intermediate mixture, empirical hazard functions as black lines. Each panel presents 4 individual participants' hazard functions from the second existence proof, except for the third row, which contains three hazard functions. The left-most column of panels depicts the omnibus hazard functions for cocktail mixtures corresponding to empirical distributions on the same row. The right-most column depicts hazard functions of corresponding omnibus ex-Gaussian distributions. The plots make clear that cocktail simulations better approximate empirical hazard functions and that ex-Gaussian hazard functions fail to display the prominent peaks that are present in both empirical and cocktail mixture hazard functions.

response time distributions, as indexed by the wider range of simulation parameters required to capture empirical distributions. Nonetheless, no new assumptions were required to accommodate the more inclusive dispersion of pronunciation times. Once again, different mixes of power-law and lognormal data points accommodated individual differences in the pattern of dispersion.

In both existence proofs, power-law and lognormal behavior sufficiently mimicked the three characteristic hazard functions that distinguish individual participants. This success is most important. 
It guarantees generality beyond these particular existence proofs; the same hazard functions are found in most or all response time performances. This outcome further justifies the choice of wordnaming times as a test case. What we have learned from the particulars of pronunciation-time distributions tells us about general system properties of cognition.

We have learned, for instance, that response time distributions vary qualitatively across participants, even in the same cognitive task. Thus, one must either posit different processes for the different participants or common processes that can change the quality of their dynamics. This has wide-ranging implications for how to think about human performance, whether the goal is data analysis of a particular experiment or a comprehensive theory of cognitive activity.

One challenge yet remains to the reliability of cocktail simulations, however, which we fully discuss now. The possibility exists, at least in principle, that our fitting technique, with six parameters, is too flexible and simply exploits ad hoc statistical mimicking. This is a potential problem for any statistical description of any empirical pattern. As we noted earlier in this article, no theory has ironclad protection from this challenge. Any curve, including hazard functions, can be mimicked in many ways, and models with more parameters are more dubious than models with fewer parameters.

It is worth asking, for example, whether conclusions based on hazard function structures, such as peaks, are trustworthy. As one progresses further into the right tail of a hazard function, for instance, the statistical reliability of the hazard function tends to diminish. Events that unfold in the slow tails of response time distributions are always more sparsely sampled than events that unfold near the mode. Other statistical descriptions of response time are also affected by this limitation, not only hazard functions, and some statistical tools exist that do a better job than others to compensate for these issues.

We addressed the previous concern with the following statistical tools. We chose kernel density estimation (Silverman, 1989; Van Zandt, 2000) and the Miller and Singpurwalla (1980) hazard function estimation routine. In addition, we combined the density and hazard estimation tools with bootstrapping procedures. Bootstrapping makes the inherent variability of the statistical tools visible by repeatedly resampling simulated distributions to evaluate them in the same manner as empirical distributions.

Bootstrapping yields visible confidence intervals across the estimated function, which supplies information about the reliability of the features of simulated distributions. For example, the synthetic cocktail mixtures typically preserved the peaks and other prominent features of empirical hazard functions. Also, reports of other researchers using different tasks buttress, or focus on, these features of hazard functions. The three characteristic hazard shapes were established in the literature at least as early as Luce's (1986) monograph, and they replicate across tasks and laboratories.

Laboratory methods were also chosen to enhance statistical reliability. We collected 1,100 observations from each individual to greatly increase the resolution of our analyses and used single laboratory sessions to minimize parameter variability across different sessions. Very importantly, all parameter estimation and all distribution fitting were executed for individual participants to avoid known artifacts that result from collapsing across participants (e.g., Estes \& Maddox, 2005).
Nevertheless, all the previous safeguards to enhance reliability can fail to protect curve fitting from an infinite regress of possible fits. In that regard, Van Zandt and Ratcliff (1995) recommended strictly coherent theoretical motivation to justify the chosen description. They placed a premium on principled, theoretically motivated, a priori choices when adopting statistical descriptions. Thus, models must be grounded in reasonable theoretical assumptions, which can be tested with empirical patterns.

The cocktail simulations were strictly motivated by interaction dominant dynamics, which previously anticipated widely observed, fractal, 1/f scaling. Power-law and lognormal distributions are both necessary consequences of multiplicative interactions, but neither of these choices is obvious outside of a theory based on interaction dominant dynamics. $1 / \mathrm{f}$ scaling by itself, for instance, does not strongly constrain the choice of event-time distribution. The default distribution of synthetic 1/f noise is the Gaussian distribution.

$1 / \mathrm{f}$ scaling only entails interdependent processes and multiplicative interactions within a theory in which processes interact via interaction dominant dynamics, which of course also predicts inverse power-law dispersion (Bak, 1996; Jensen, 1998; Montroll \& Shlesinger, 1982). The remaining challenge was to invent a reasonable scheme to combine power-law and lognormal distributions. We chose the simplest scheme that we imagined. The six parameters of the cocktail mixtures come simply from locating both distributions within an interval on the response time axis and mixing them in varying proportions.

In sum, the cocktail hypothesis comes from the a priori hypothesis of interaction dominant dynamics, and cocktail simulations supply independent tests of this inclusive hypothesis (cf. Wagenmakers, Farrell, \& Ratcliff, 2005). Consequently, the cocktail hypothesis complies with Van Zandt and Ratcliff's (1995) recommendations to minimize ad hoc statistical mimicking.

\section{General Discussion}

Our essential finding is that the synthetic cocktail mixtures, symptomatic of interdependence and multiplicative interaction, successfully mimicked empirical pronunciation-time probability density and hazard functions. Successfully means simply that the cocktail descriptions were not contradicted statistically by any of the analyses we conducted. All other models that we examined were less successful.

Shapes of synthetic cocktail density functions were consistent with the shapes of empirical probability density functions over large portions of the response time interval, whether plotted on linear or double-logarithmic axes. The cocktail mixtures reliably produced cumulative distribution functions that passed a Kolmogorov-Smirnov test of equivalence. Also, the cocktail mixtures captured the qualitative features of individual participants' hazard functions, including the three shapes that characterize most response time data documented in the literature.

The specific value of the power-law exponent and the relative mixture proportions in cocktails simulate the transition from power-law dominant to lognormal dominant densities. Empirical transitions presumably reflect tipping points or critical points of constraint that transition individual word pronunciations into tightly reinforced dynamics, in the broad sense of reinforced, and therein minimize the contingencies of feedback. This hypothesis 
marks constraints and the power-law-lognormal trade-off to account for individual differences.

Cocktail mixtures also closely replicate the values of, and relationships among, empirical parameters such as mean, standard deviation, and skew. In sum, a working hypothesis grounded in the interaction dominant dynamics of feedback processes captures most of the empirical facts of individual participants' pronunciation-time distributions. We are not aware of a more inclusive or more general account of response time data.

\section{Standard Cognitive Manipulations}

What of the vast library of standard mean effects that resulted from standard cognitive manipulations? How do they make contact with the present success? Individual differences in background history, development, current status, and context-in currently available constraints-were predicted to yield different mixes of two kinds of multiplicative functions. Systematic qualitative differences among the consequent hazard functions corroborated the prediction. Reliable qualitative differences in data from the same word-naming task, however, bring into question the logic of design or analysis that contrasts or equates descriptive statistics from different college students' response times. Such contrasts are not ruled out by this finding, but it raises the bar for their justification. One way forward is to develop new statistical tools that are commensurate with qualitative variability changes and interaction dominant dynamics (e.g., Riley \& Van Orden, 2005).

Also, we noted several times how, all other things being equal, adequate accounting of distributions or densities supplies the more inclusive account. For instance, how can an account based on means alone anticipate correlations among means, standard deviations, and skew? Likewise, how can an account based on distributions alone anticipate the three characteristic hazard functions? More narrowly conceived empirical findings are not necessarily inconsistent with the present results, but they are weaker tests of models. Nonetheless, if researchers could expand the existing library and reexamine all mean effects using contrasts among densities and hazard functions, it would greatly increase empirical power to constrain models and theories.

For example, standard manipulations usually test stimulus properties in performance, which define different word classes. A general basis of many word-class distinctions is ambiguity. Ambiguity refers to the relative consistency of relations between spelling patterns and pronunciations, among other things; consider _ear in pear versus near. Ambiguous spellings support more than one pronunciation, and ambiguous words are more slowly pronounced on average in a word-naming task (Glushko, 1979).

Relatively more ambiguous words also disperse their pronunciation times more broadly, in harmony with the present account (Holden, 2002). Specifically, word items can be more or less infected by ambiguity; that is, ambiguity can be restricted locally to a few letters or the whole word's spelling can be ambiguous as in homographs such as tear and wind (Van Orden \& Kloos, 2005, is a review). A close look at word-class effects, using a systematic increase in pronunciation ambiguity (e.g., punt vs. pear vs. tear), sampling across participants, revealed progressively wider dispersion in the heavy slow tails of pronunciation-time distributions.

The more all-embracing the infection of ambiguity, the more prominent the power-law behavior in the slow tail. Yet the fast leading edges of contrasted pronunciation distributions, up to the mode, were close to the same. The same pattern was found for ambiguity in a lexical decision task using patterns of pronunciation that support multiple spellings, such as cube versus hone versus hare (Holden, 2002; cf. Stone et al., 1997). In fact, the shapes of the response time distributions to different item classes in Holden (2002) appear consistent with the cocktail mixtures (however, the sample sizes were smaller than those reported here). We reexamined those distributions to determine whether different item classes yielded different hazard functions. Indeed, the item classes most infected by ambiguity yielded hazard functions most like the power-law dominant mixtures in both word naming and lexical decision. The item classes least infected with ambiguity yielded hazard functions most like the lognormal dominant mixtures. Qualitatively similar dispersion effects have been reported in other word-recognition studies (e.g., Andrews \& Heathcote, 2001; Balota et al., 2004).

Power-law behavior was reported in other reading tasks as well. Schultz and Tabor (2005) reported power-law behavior in sentence reading times. The extent of garden-path ambiguity in sentence comprehension amplifies power-law behavior in the slow heavy tails of reading time distributions. As in Holden (2002), the extent of power-law behavior is determined by word or phrase ambiguity. These outcomes agree with the present studies, and the hypothesis that motivates the trade-off between power-law and lognormal behavior. All other things being equal, relatively ambiguous stimuli produce power-law behavior, and sufficiently unambiguous stimuli produce lognormal behavior. Unambiguous in our terms refers to available constraints sufficient to mask, or dampen down, the contingencies of feedback (cf. Gottlob, Goldinger, Stone, \& Van Orden, 1999; Van Orden \& Goldinger, 1994).

The success of the cocktail mixtures generalizes to response time data from other cognitive tasks as well (e.g., Ashby et al., 1993; Balakrishnan \& Ashby, 1992; Luce, 1986; Maddox et al., 1998). Thus, the manner in which cognitive manipulations impact the shape of response time distributions may likewise generalize and give a common account of contrasts between items, as well as other factorial contrasts.

\section{Constraint and Cognitive Performance}

Historically, cognitive manipulations have been equated with cognitive components, as efficient causes, in causal chains that unfold within the brief interval of a response time. Causal configurations as constraints, however, refer to relations among processes and the coordination of their activities in nonlinear dynamics (Juarrero, 1999). Constraints refer to any aspect of history, context, present status of mind and body, and task environment that limit or control the degrees of freedom for cognition and behavior.

Many standard causal manipulations can be reinterpreted as manipulations of available constraints. Consequently, one can generate predictions and manipulate the dispersion of response times. Other things being equal, manipulations that entail stronger competitions among contradictory sources of constraint or that rely on a weaker arrays of constraints should display more widely dispersed behavior, the increasing presence of power-law behavior.

The current existence proofs demonstrate the plausibility of constraints on performances organized via interaction dominant 
dynamics. From a cognitive perspective, supporting constraints change slowly, more slowly than the pace of trial performances, while they dispose the mind and body on each trial to receive and pronounce the stimulus word (Hollis et al., 2009; Van Orden, Holden, \& Turvey, 2003). The arrival of the stimulus further narrows degrees of freedom, to close the system and unfold the specific pronunciation response (Järvilehto, 1998).

From a physiological perspective, constraints on interaction dominant dynamics, within the trial, coordinate the fastest rhythms of the nervous system with the stimulus perturbation of the environment upward through intermediate timescales of brain and body to the limiting timescale of performance, the timescale on which the stimulus word's pronunciation unfolds (compare Buzsáki, 2006). Prestimulus preparation and coordination of embodied dynamics, across all the body's timescales, guarantee the appropriate poststimulus degrees of freedom for within-trial dynamics on their very fast timescales (Gilden, 2001; Hollis et al., 2009).

\section{Conclusions}

Interaction dominant dynamics anticipated, previously, the widely evident $1 / \mathrm{f}$ scaling in trial series of response times. Presently, interaction dominant dynamics successfully predict that aggregate response times disperse as power laws, due to interdependence among feedback processes. Interaction dominant dynamics as multiplicative feedback processes also predict power-law behavior, as well as lognormal behavior. Power-law and lognormal dispersion is symptomatic of multiplicative interactions among process on a hierarchy of timescales.

If mixtures of power-law and lognormal distributions mimic empirical dispersion, then empirical dispersion originates in a vast array of multiplicative interactions. This conclusion rests upon an inverse inference, similar to the classic inference trusted for Gaussian dispersion. Strictly speaking, then, the success of the cocktail mixtures is not sufficient evidence of interaction dominant dynamics: Success is simply a necessary consequence. That is what it means when we call cocktail simulations existence proofs. In this way, of course, the present support in evidence is like most evidence in science.

The complex emergence of online word-naming performance, or any response behavior, presents daunting challenges without simplifying principles. In that regard, all sources of constraint combine via multiplicative interaction. Each trial performance is a temporary dynamical structure visible from above in the slower timescale patterns of scaling relations and from below in the faster timescale changes that dispersion portrays.

Still, coordination across all these timescales is necessary to perform a word pronunciation, so scaling relations, inverse power laws, and lognormal dispersion must imply complementary mechanisms. In fact, it suffices to speak of one kind of mechanism, interaction dominant dynamics, which coordinates processes slower than and faster than the trial pace of measurement. We draw this essential conclusion from the success of the present existence proofs.

\section{References}

Anderson, C. (2006). The long tail: Why the future of business is selling less of more. New York: Hyperion.
Andrews, S., \& Heathcote, A. (2001). Distinguishing common and taskspecific processes in word identification: A matter of some moment? Journal of Experimental Psychology: Learning, Memory, and Cognition, 27, 514-544.

Ashby, F. G., Tien, J.-Y., \& Balakrishnan, J. D. (1993). Response time distributions in memory scanning. Journal of Mathematical Psychology, $37,526-555$.

Bak, P. (1996). How nature works. New York: Springer-Verlag.

Balakrishnan, J. D., \& Ashby, F. G. (1992). Subitizing: Magical numbers or mere superstition? Psychological Research, 54, 80-90.

Balota, D. A., Cortese, M. J., Sergent-Marshall, S. D., Spieler, D. H., \& Yap, M. J. (2004). Visual word recognition of single-syllable words. Journal of Experimental Psychology: General, 133, 283-316.

Balota, D. A., \& Spieler, D. H. (1999). Frequency, repetition, and lexicality effects in word recognition: Beyond measures of central tendency. Journal of Experimental Psychology: General, 128, 32-55.

Buzsáki, G. (2006). Rhythms of the brain. New York: Oxford University Press.

Chechile, R. A. (2003). Mathematical tools for hazard function analysis. Journal of Mathematical Psychology, 42, 432-471.

Clauset, A., Shalizi, C. R., \& Newman, M. E. J. (2007). Power-law distributions in empirical data. E-print: arXiv:0706.1062v1 [physics.data-an], retrieved December 18, 2008, from http://arxiv.org/ abs/0706.1602

Colangelo, A., Holden, J. G., Buchanan, L., \& Van Orden, G. C. (2004). Speculation about behavior, brain damage, and self-organization: The other way to herd a cat. Brain and Language, 90, 151-159.

Depew, D. J., \& Weber, B. H. (1997). Darwinism evolving: System dynamics and the genealogy of natural selection. Cambridge, MA: MIT Press.

Efron, B., \& Tibshirani, R. J. (1993). An introduction to the bootstrap. New York: Chapman \& Hall.

Estes, W. K., \& Maddox, W. T. (2005). Risks of drawing inferences about cognitive processes from model fits from individual versus average performance. Psychonomic Bulletin \& Review, 12, 403-408.

Farmer, J. D. (1990). A Rosetta stone for connectionism. Physica D: Nonlinear Phenomena, 42, 153-187.

Farmer, J. D., \& Geanakoplos, J. (2005). Power laws in economics and elsewhere. Chapter from preliminary draft of book entitled Beyond equilibrium and efficiency, retrieved September 19, 2008, from http:// www.santafe.edu/ jdf/papers/powerlaw3.pdf

Faust, M. E., Balota, D. A., Spieler, D. H., \& Ferraro, F. R. (1999). Individual differences in information-processing rate and amount: Implications for group differences in response latency. Psychological Bulletin, 125, 777-799.

Forster, K. I., \& Forster, J. C. (1996). DMASTR display system for mental chronometry (Version 5.18) [Computer software]. Tucson, AZ: Author.

Furusawa, C., Suzuki, T., Kashiwagi, A., Yomo, T., \& Kaneko, K. (2005). Ubiquity of log-normal distributions in intra-cellular reaction dynamics. Biophysics, 1, 25-31.

Gilden, D. L. (2001). Cognitive emissions of 1/f noise. Psychological Review, 108, 33-56.

Glushko, R. J. (1979). The organization and activation of orthographic knowledge in reading aloud. Journal of Experimental Psychology: Human Perception and Performance, 5, 674-691.

Gnedenko, B. V., \& Khinchin, A. Y. (1962). An elementary introduction to the theory of probability (L. Boron, Trans.). New York: Dover.

Gottlob, L. R., Goldinger, S. D., Stone, G. O., \& Van Orden, G. C. (1999). Reading homographs: Orthographic, phonologic, and semantic dynamics. Journal of Experimental Psychology: Human Perception and Performance, 25, 561-574.

Hale, S., Myerson, J., Faust, M. E., \& Fristoe, N. (1995). Converging evidence for domain-specific slowing from multiple nonlexical tasks and 
multiple analytic methods. Journals of Gerontology: Psychological Sciences, 50(B), P202-P211.

Heathcote, A., Popiel, S. J., \& Mewhort, D. J. K. (1991). Analysis of response time distributions: An example using the Stroop task. Psychological Bulletin, 109, 340-347.

Holden, J. G. (2002). Fractal characteristics of response time variability. Ecological Psychology, 14, 53-86.

Holden, J. G. (2005). Gauging the fractal dimension of response times from cognitive tasks. In M. A. Riley \& G. C. Van Orden (Eds.), Contemporary nonlinear methods for behavioral scientists: A Webbook tutorial (pp. 267-318). Retrieved April 8, 2005, from http://www.nsf.gov/sbe/ bcs/pac/nmbs/nmbs.jsp

Hollis, G., Kloos, H., \& Van Orden, G. C. (2009). Origins of order in cognitive activity. In S. Guastello, M. Koopmans, \& D. Pincus (Eds.), Chaos and complexity in psychology: The theory of nonlinear dynamical systems (pp. 206-241). New York: Cambridge University Press.

Järvilehto, T. (1998). The theory of the organism-environment system: I. Description of the theory. Integrative Physiological and Behavioral Science, 33, 321-334.

Jensen, H. J. (1998). Self-organized criticality. Cambridge, England: Cambridge University Press.

Jones, B. K. (2002). Logarithmic distributions in reliability analysis. Microelectronics Reliability, 42, 779-786.

Juarrero, A. (1999). Dynamics in action. Cambridge, MA: MIT Press.

Kello, C. T., Anderson, G. G., Holden, J. G., \& Van Orden, G. C. (2008). The pervasiveness of $1 / \mathrm{f}$ scaling in speech reflects the metastable basis of cognition. Cognitive Science, 32, 1-15.

Kello, C. T., Beltz, B. C., Holden, J. G., \& Van Orden, G. C. (2007). The emergent coordination of cognitive function. Journal of Experimental Psychology: General, 136, 551-568.

Kirchner, J. W., Hooper, R. P., Kendall, C., Neal, C., \& Leavesley, G. (1996). Testing and validating environmental models. Science of the Total Environment, 183, 33-47.

Klein, J. L. (1997). Statistical visions in time: A history of time series analysis 1662-1938. Cambridge, England: Cambridge University Press.

Kuçera, H., \& Francis, W. (1967). Computational analysis of present-day American English. Providence, RI: Brown University Press.

Kugler, P. N., \& Turvey, M. T. (1987). Information, natural law, and the self-assembly of rhythmic movement. Hillsdale, NJ: Erlbaum.

Lewontin, R. C. (1974). The analysis of variance and the analysis of causes. American Journal of Human Genetics, 26, 400-411.

Limpert, E., Stahel, W. A., \& Abbt, M. (2001). Log-normal distributions across science: Keys and clues. BioScience, 51, 341-352.

Luce, R. D. (1986). Response times: Their role in inferring elementary mental organization. New York: Oxford University Press.

Maddox, W. T., Ashby, F. G., \& Gottlob, L. R. (1998). Response time distributions in multidimensional perceptual categorization. Perception \& Psychophysics, 60, 620-637.

Miller, D. R., \& Singpurwalla, N. D. (1980). Failure rate estimation using random smoothing. Sankhya, 42, 217-228.

Mitzenmacher, M. (2003). A brief history of generative models for power law and lognormal distributions. Internet Mathematics, 1, 226-251.

Molenaar, P. C. M. (2008). On the implications of the classical ergodic theorems: Analysis of developmental processes has to focus on intraindividual variation. Developmental Psychobiology, 50, 60-69.

Montroll, E. W., \& Shlesinger, M. F. (1982). On 1/f noise and other distributions with long tails. Proceedings of the National Academy of Sciences, USA, 79, 3380-3383.

Moreno, M. A. (2002). A nonlinear dynamical systems perspective on response time distributions. Doctoral dissertation, Arizona State University.

Moreno, M. A., Buchanan, L., \& Van Orden, G. C. (2002). Variability in aphasic patients' response times. Brain \& Cognition, 48, 469-474.
Newman, M. E. J. (2005). Power laws, Pareto distributions and Zipf's law. Contemporary Physics, 46, 323-351.

Peng, C. K., Havlin, S., Stanley, H. E., \& Goldberger, A. L. (1995). Quantification of scaling exponents and crossover phenomena in nonstationary heartbeat time series. Chaos, 5, 82-87.

Philippe, P. (2000). Epidemiology and self-organized critical systems: An analysis in waiting times and disease heterogeneity. Nonlinear Dynamics, Psychology, and Life Sciences, 4, 275-295.

Porter, T. M. (1986). The rise of statistical thinking 1820-1900. Princeton, NJ: Princeton University Press.

Ratcliff, R. (1979). Group reaction time distributions and an analysis of distribution statistics. Psychological Bulletin, 86, 446-461.

Riley, M. A., \& Turvey, M. T. (2002). Variability and determinism in elementary behaviors. Journal of Motor Behavior, 34, 99-125.

Riley, M. A., \& Van Orden, G. C. (2005). Tutorials in contemporary nonlinear methods for the behavioral sciences. Retrieved April 8, 2005, from http://www.nsf.gov/sbe/bcs/pac/nmbs/nmbs.jsp

Schmiedek, F., Oberauer, F., Wilhelm, W. W., Süß, H.-M., \& Wittmann, W. W. (2007). Individual differences in components of reaction time distributions and their relations to working memory and intelligence. Journal of Experimental Psychology: General, 136, 414-429.

Schultz, A., \& Tabor, W. (2005). Reaction time distributions in sentence processing. In L. D. Rosenblum (Ed.), Proceedings of the 13th International Conference on Perception and Action, Pacific Grove, CA (p. 49). Riverside: University of California.

Schwarz, W. (2001). The ex-Wald distribution as a descriptive model of response times. Behavior Research Methods, Instruments, and Computers, 33, 457-469.

Silverman, B. W. (1989). Density estimation for statistics and data analysis. London: Chapman \& Hall.

Speelman, C. P., \& Kirshner, K. (2005). Beyond the learning curve: The construction of mind. Oxford, England: Oxford University Press.

Spieler, D. H., \& Balota, D. A. (1997). Bringing computational models of word naming down to the item level. Psychological Science, 8, 411416.

Sternberg, S. (1969). The discovery of processing stages: Extensions of Donders' method. Acta Psychologica, 30, 276-315.

Stigler, S. M. (1986). The history of statistics: The measurement of uncertainty before 1900. Cambridge, MA: Harvard University Press.

Stone, G. O., Vanhoy, M., \& Van Orden, G. C. (1997). Perception is a two-way street: Feedforward and feedback phonology in visual word recognition. Journal of Memory and Language, 36, 337-359.

Tankard, J. W. (1986). The statistical pioneers. Cambridge, MA: Schenkman Publishing Company.

Theiler, J., Eubank, S., Longtin, A., Galdrikian, B., \& Farmer, J. D. (1992). Testing for nonlinearity in time series: The method of surrogate data. Physica D: Nonlinear Phenomena, 58, 77-94.

Thornton, T. L., \& Gilden, D. L. (2005). Provenance of correlations in psychological data. Psychonomic Bulletin \& Review, 12, 409-441.

Townsend, J. T. (1990). Truth and consequences of ordinal differences in statistical distributions: Toward a theory of hierarchical inference. Psychological Bulletin, 108, 551-567.

Turvey, M. T., \& Moreno, M. A. (2006). Physical metaphors for the mental lexicon. The Mental Lexicon, 1, 7-33.

Van Orden, G. C. (2007, February). The fractal picture of health and wellbeing. Psychological Science Agenda, 21(2). Retrieved December 18, 2008, from http://www.apa.org/science/psa/vanorden.html

Van Orden, G. C., \& Goldinger, S. D. (1994). Interdependence of form and function in cognitive systems explains perception of printed words. Journal of Experimental Psychology: Human Perception and Performance, 20, 1269-1291.

Van Orden, G. C., Holden, J. G., \& Turvey, M. T. (2003). Selforganization of cognitive performance. Journal of Experimental Psychology: General, 132, 331-350. 
Van Orden, G. C., Holden, J. G., \& Turvey, M. T. (2005). Human cognition and 1/f scaling. Journal of Experimental Psychology: General, $134,117-123$.

Van Orden, G. C., Kello, C. T., \& Holden, J. G. (in press). Situated behavior and the place of measurement in psychological theory. Ecological Psychology.

Van Orden, G. C., \& Kloos, H. (2005). The question of phonology and reading. In M. J. Snowling \& C. Hulme (Eds.), The science of reading: A handbook (pp. 61-78). Oxford, England: Blackwell Publishers.

Van Orden, G. C., Moreno, M. A., \& Holden, J. G. (2003). A proper metaphysics for cognitive performance. Nonlinear Dynamics, Psychology, and Life Sciences, 1, 47-58.

Van Orden, G. C., Pennington, B. F., \& Stone, G. O. (2001). What do double dissociations prove? Cognitive Science, 25, 111-172.

Van Zandt, T. (2000). How to fit a response time distribution. Psychonomic Bulletin \& Review, 7, 424-465.

Van Zandt, T. (2002). Analysis of response time distributions. In J. T. Wixted (Vol. Ed.) \& H. Pashler (Series Ed.), Stevens' handbook of experimental psychology: Vol. 4. Methodology in experimental psychology (3rd ed., pp. 461-516). New York: Wiley.

Van Zandt, T. (2003). On the use of the ex-Gaussian distribution for characterizing response times. Unpublished manuscript.

Van Zandt, T., \& Ratcliff, R. (1995). Statistical mimicking of reaction time data: Single process models, parameter variability, and mixtures. Psychonomic Bulletin \& Review, 2, 20-54.

Wagenmakers, E.-J. (in press). Methodological and empirical develop- ments for the diffusion model of response time and accuracy. European Journal of Cognitive Psychology.

Wagenmakers, E.-J., \& Brown, S. (2007). On the relation between the mean and standard deviation of a response time distribution. Psychological Review, 114, 830-841.

Wagenmakers, E.-J., Farrell, S., \& Ratcliff, R. (2005). Human cognition and a pile of sand: A discussion of serial correlations and self-organized criticality. Journal of Experimental Psychology: General, 134, 108-116.

Warren, W. H. (2006). The dynamics of perception and action. Psychological Review, 113, 358-389.

West, B. J. (2006). Where medicine went wrong: Rediscovering the path to complexity. Hackensack, NJ: World Scientific.

West, B. J., \& Deering, B. (1995). The lure of modern science: Fractal thinking. River Edge, NJ: World Scientific.

Wijnants, M. L., Bosman, A. M. T., Hasselman, F., Cox, R. F. A., \& Van Orden, G. C. (2009). 1/f scaling fluctuation in movement time changes with practice in precision aiming. Nonlinear Dynamics, Psychology, and Life Sciences, 13, 79-98.

Yap, M. J., Balota, D. A., Tse, C.-S., \& Besner, D. (2008). On the additive effects of stimulus quality and word frequency in lexical decision: Evidence for opposing interactive influences revealed by RT distributional analyses. Journal of Experimental Psychology: Learning, Memory, and Cognition, 34, 495-513.

Received December 19, 2006 Revision received October 16, 2008 Accepted October 20, 2008

\section{ORDER FORM}

Start my 2009 subscription to Psychological Review ISSN: 0033-295X

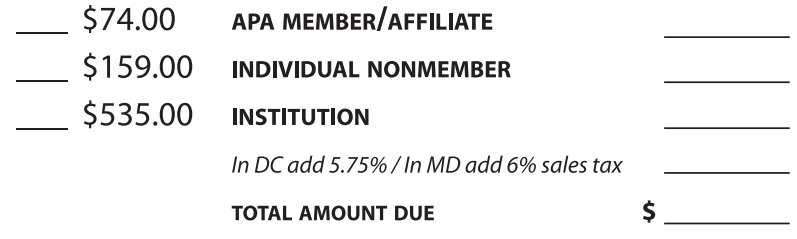

Subscription orders must be prepaid. Subscriptions are on a calendar year basis only. Allow $4-6$ weeks for delivery of the first issue. Call for international subscription rates.

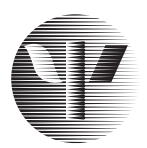

SEND THIS ORDER FORM TO

American Psychological Association

Subscriptions

750 First Street, NE

AMERICAN

ASSOCIATION
Check enclosed (make payable to APA)

Charge my: $\square$ Visa $\square$ MasterCard $\square$ American Express

Cardholder Name

Card No. _ Exp. Date

Signature (Required for Charge)

\section{Billing Address}

Street

City State Zip

Daytime Phone

E-mail

Mail To

Name

Address

City _ State Zip _

APA Member \#
Call 800-374-2721 or 202-336-5600

Fax 202-336-5568 :TDD/TTY 202-336-6123

For subscription information,

e-mail:subscriptions@apa.org 